\title{
THE BUBBLING GALACTIC DISK
}

\author{
E. Churchwell, ${ }^{1}$ M. S. Povich, ${ }^{1}$ D. Allen, ${ }^{1}$ M. G. Taylor, ${ }^{1}$ M. R. Meade, ${ }^{1}$ B. L. Babler,${ }^{1}$ R. Indebetouw, ${ }^{2}$ \\ C. Watson, ${ }^{3}$ B. A. Whitney, ${ }^{4}$ M. G. Wolfire, ${ }^{5}$ T. M. Bania, ${ }^{6}$ R. A. Benjamin, ${ }^{7}$ D. P. Clemens, ${ }^{6}$ \\ M. Cohen, ${ }^{8}$ C. J. Cyganowski, ${ }^{1}$ J. M. Jackson, ${ }^{6}$ H. A. Kobulnicky, ${ }^{9}$ J. S. Mathis, ${ }^{1}$ \\ E. P. Mercer, ${ }^{6}$ S. R. Stolovy, ${ }^{10}$ B. Uzpen, ${ }^{9}$ D. F. Watson, ${ }^{1}$ and M. J. WolfF ${ }^{4}$ \\ Received 2005 November 14; accepted 2006 June 9
}

\begin{abstract}
A visual examination of the images from the Galactic Legacy Infrared Mid-Plane Survey Extraordinaire (GLIMPSE) has revealed 322 partial and closed rings that we propose represent partially or fully enclosed three-dimensional bubbles. We argue that the bubbles are primarily formed by hot young stars in massive star formation regions. We have found an average of about 1.5 bubbles per square degree. About $25 \%$ of the bubbles coincide with known radio $\mathrm{H}$ II regions, and about 13\% enclose known star clusters. It appears that B4-B9 stars (too cool to produce detectable radio $\mathrm{H}$ II regions) probably produce about three-quarters of the bubbles in our sample, and the remainder are produced by young O-B3 stars that produce detectable radio $\mathrm{H}$ in regions. Some of the bubbles may be the outer edges of $\mathrm{H}$ in regions where PAH spectral features are excited and may not be dynamically formed by stellar winds. Only three of the bubbles are identified as known SNRs. No bubbles coincide with known planetary nebulae or W-R stars in the GLIMPSE survey area. The bubbles are small. The distribution of angular diameters peaks between $1^{\prime}$ and $3^{\prime}$ with over $98 \%$ having angular diameters less than $10^{\prime}$ and $88 \%$ less than $4^{\prime}$. Almost $90 \%$ have shell thicknesses between 0.2 and 0.4 of their outer radii. Bubble shell thickness increases approximately linearly with shell radius. The eccentricities are rather large, peaking between 0.6 and 0.7 ; about $65 \%$ have eccentricities between 0.55 and 0.85 .
\end{abstract}

Subject headings: H II regions — ISM: bubbles

Online material: extended color figure, machine-readable tables

\section{INTRODUCTION}

The Galactic Legacy Infrared Mid-Plane Survey Extraordinaire (GLIMPSE; Benjamin et al. 2003) provides a new, high spatial resolution view of the inner two-thirds of the Galactic plane at infrared wavelengths from 3.6 to $8.0 \mu \mathrm{m}$. A visual inspection of the GLIMPSE images reveals a large number of full or partial ring structures. We postulate that the rings are projections of three-dimensional shells and henceforth refer to them as bubbles. The prevalence of bubbles at mid-infrared wavelengths is not unexpected. They were a common feature in the Midcourse Space Experiment (MSX; Price et al. 2001; Cohen \& Green 2001). Large bubbles (greater than $10^{\prime}$ in diameter) have also been identified in the $\mathrm{H}_{\mathrm{I}}$ Southern Galactic Plane Survey (McClure-Griffiths et al. 2002) and with the Wisconsin H $\alpha$ Mapper (WHAM; Haffner et al. 2003). The new GLIMPSE images provide $\sim 10$ times higher

\footnotetext{
1 Department of Astronomy, University of Wisconsin at Madison, 475 North Charter Street, Madison, WI 53706.

2 Department of Astronomy, University of Virginia, P.O. Box 3818, Charlottesville, VA 22903-0818.

3 Department of Physics, Manchester College, North Manchester, IN 46962.

4 University of Colorado, Space Science Institute, 1540 30th Street, Suite 23, Boulder, CO 80303-1012.

5 Department of Astronomy, University of Maryland, College Park, MD 20742-2421.

${ }^{6}$ Institute for Astrophysical Research, Boston University, 725 Commonwealth Avenue, Boston, MA 02215.

7 Department of Physics, University of Wisconsin at Whitewater, 800 West Main Street, Whitewater, WI 53190.

8 Radio Astronomy Laboratory, University of California, 601 Campbell Hall, Berkeley, CA 94720.

9 Department of Physics and Astronomy, University of Wyoming, Laramie, WY 82071.

${ }^{10}$ Spitzer Science Center, California Institute of Technology, MC 314-6, 1200 East California Boulevard, Pasadena, CA 91125.
}

spatial resolution and $\sim 100$ times better sensitivity than $M S X$, permitting detection of smaller and fainter bubbles than $M S X$ or any other Galactic plane survey to date.

Bubbles are important morphological features in the interstellar medium (ISM). The study of bubbles gives information about the stellar winds that produce them and the structure and physical properties of the ambient ISM into which they are expanding. Additional physical insights include the hydrodynamics of gas and dust in expanding bubbles, the impact of expanding bubbles on magnetic fields in the diffuse ISM, and mass-loss rates during the evolution of stars. Dynamically formed bubbles that are bright at mid-infrared wavelengths but not coincident with supernova remnants (SNRs) or planetary nebulae require a star or cluster with enough UV emission to excite the polycyclic aromatic hydrocarbon (PAH) features in the IRAC 5.8 and $8.0 \mu \mathrm{m}$ bands and a strong enough wind to evacuate a dust-free cavity. Some bubbles exhibit morphologies suggestive of triggered or stimulated star formation. Star formation could be triggered by the expansion of a bubble when the leading shock front overruns and compresses a preexisting molecular cloud core, resulting in gravitational collapse of the core. Bubbles that exhibit this star formation mechanism provide a crucial path for further understanding how triggered star formation works.

We have identified candidate bubbles in the GLIMPSE survey and have measured their general morphological properties. We investigate the frequency distributions of morphological properties and their variations with position in the Galaxy. These statistical data will provide a basis for understanding the physics of bubble formation and the interactions of young stars with their environments. We divide the GLIMPSE survey area by longitude into northern $\left(l=10^{\circ}-65^{\circ}\right)$ and southern $\left(l=295^{\circ}-350^{\circ}\right)$ regions to facilitate comparison of possible morphological, positional, and size differences. Previous radio continuum, $\mathrm{H} \mathrm{I}$, and 
CO surveys (see following sections) have shown some puzzling systematic differences between the northern and southern plane. We show in the following that some of the same asymmetries are also found in the distribution of infrared bubbles. In $\S 2$ we describe the data and the techniques used to determine morphological properties. In $\S 3$ we report the apparent distribution of bubbles in the inner Galaxy and bubble morphological properties, and we identify different types of known objects that produce the bubbles. We present distances and physical sizes of those bubbles that have associated $\mathrm{H}$ II regions in $\S 4$. We explore a possible link between multiple bubble complexes and triggered star formation in $\S 5$. Finally, our results and conclusions are summarized in $\oint 6$.

\section{THE DATA}

High-resolution ( $1^{\prime \prime} .2$ pixels) image mosaics $\left(l \times b=3^{\circ} \times 2^{\circ}\right)$ were used to search for bubbles over the entire GLIMPSE survey area. The mosaics were created by the GLIMPSE pipeline after image artifacts such as cosmic rays, stray light, column pulldown, and banding were removed. ${ }^{11}$ The SSC Mopex package was used to remove cosmic rays, and the IPAC Montage package was used to mosaic the images. The angular resolutions of the Infrared Array Camera (IRAC; Fazio et al. 2004) on the Spitzer Space Telescope (Werner et al. 2004) range from $\sim 1 " .5$ at $3.6 \mu \mathrm{m}$ to $\sim 1 " .9$ at $8.0 \mu \mathrm{m}$. IRAC bands 2 (4.5 $\mu \mathrm{m}$ : blue $), 3$ (5.8 $\mu \mathrm{m}$ : green $)$, and $4(8.0 \mu \mathrm{m}$ : red $)$ were viewed in false colors using the display program ds $9^{12}$ with color ratios adjusted such that the bubbles stood out above the general background emission. These images were then visually searched for partial and complete ring structures. Because specification of morphology is subjective and prone to individual biases, we have provided an online four-color $(3.6 \mu \mathrm{m}$ : blue $; .5 \mu \mathrm{m}$ : green; $5.8 \mu \mathrm{m}$ : orange $; 8.0 \mu \mathrm{m}$ : red ) image archive ${ }^{13}$ that contains images of each bubble in our catalog. We recommend referring to this archive for bubbles of specific interest.

The "elliptical annulus" feature in ds9 was used to estimate the approximate center position and the semimajor and semiminor axes of the inner and outer ellipses.

\subsection{Bubble Selection Effects}

The catalog of bubbles given here is incomplete due to several selection effects that conspire to underestimate the true number of bubbles in the GLIMPSE survey area. (1) Nearby bubbles are favored because distant ones are masked by foreground emission in the Galactic plane. This implies that bubbles at the near kinematic distance are more likely to be detected than those at the far kinematic distance. (2) Faint bubbles are masked by bright background emission. (3) The ring morphology of small, bright bubbles is often not apparent because bright diffuse emission fills in the bubble cavities, making it difficult or impossible to recognize the ring morphology. (4) Bubbles that subtend very small and very large solid angles are easily missed in visual inspections.

Because we do not know the actual number of bubbles in the GLIMPSE survey area, it is impossible to quantify the completeness of the bubble catalog given here; however, it could easily be on the order of $50 \%$ or even less. Neither can we give a meaningful brightness limit at which the catalog can be declared complete since this depends strongly on both the spatial structure and brightness of the diffuse background emission.

\footnotetext{
11 See http://www.astro.wisc.edu/glimpse/docs.html.

12 See http://hea-www.harvard.edu/RD/ds9/ref.

13 See http://www.astro.wisc.edu/glimpse/bubbles/.
}

TABLE 1

Comparison of Measured Bubble Parameters

\begin{tabular}{|c|c|c|c|c|c|c|c|}
\hline \multirow[b]{3}{*}{ MEASUREMEnt } & \multirow{2}{*}{\multicolumn{4}{|c|}{ EXAMINER $^{a}$}} & \multicolumn{3}{|c|}{ All Examiners } \\
\hline & & & & & & Standard & \\
\hline & 1 & 2 & 3 & 4 & Mean & Deviation & $\mathrm{S} / \mathrm{N}$ \\
\hline Diameter $(\operatorname{arcmin}) \ldots \ldots \ldots . .$. & 2.85 & 2.85 & 2.93 & 2.63 & 2.82 & 0.13 & 22 \\
\hline Thickness $(\operatorname{arcmin}) . . . . . . .$. & 0.41 & 0.29 & 0.42 & 0.35 & 0.37 & 0.06 & 6 \\
\hline Eccentricity ....................... & 0.59 & 0.72 & 0.57 & 0.50 & 0.60 & 0.09 & 7 \\
\hline Thickness/radius............. & 0.25 & 0.20 & 0.26 & 0.23 & 0.24 & 0.03 & 9 \\
\hline
\end{tabular}

a The values given here represent the medians of the measurements of the 21 bubbles found by all four examiners.

\subsection{Observer-dependent Selection and Measurement Biases}

The bubbles in our sample were visually selected by one of the authors. However, four of the authors independently examined five $3^{\circ} \times 2^{\circ}$ mosaic images selected to span the range of longitudes from the innermost to outermost limits of the GLIMPSE survey; this included regions of high, low, and intermediate spatially variable background. The bubbles identified in these five common regions by each individual were used to estimate selection biases and the dispersion of geometrical parameters measured by multiple examiners.

In the five common regions, 21 bubbles were identified by all four examiners. This represents $40 \%$ of the bubbles found in these five $3^{\circ} \times 2^{\circ}$ mosaic images. Clear selection biases set apart the four examiners. One focused more on small bubbles, one selected more large bubbles, and the other two were generally between the two extremes. The median values of the distributions of geometrical parameters (diameter, thickness, eccentricity, and thickness/ radius) measured by each examiner for the 21 bubbles identified by all four examiners are given in Table 1 . The values for geometrical parameters given in the bubble catalog (Tables 2 and 3) are those measured by examiner 1 , who examined the entire survey. The measurements of examiner 1 were found to lie near the average of the four examiners.

Identifying bubbles and measuring their parameters is an inherently subjective process. Table 1 shows that the dispersion of geometrical parameters for the 21 bubbles measured by all four examiners ranges from $\sim 10 \%$ for the bubble diameters to $\sim 35 \%$ for the thicknesses and eccentricities. This suggests that the geometrical parameters given in the catalog are likely to be reliable at about these levels. One exception is the case of orientations of the bubble major axes. Biases introduced by the procedure of fitting elliptical annuli to images in ds 9 make orientation measurements unreliable; hence, values for bubble orientations are not included in Tables 2 and 3.

In Figures $1 a$ and $1 b$ we show two bubble images that have been fitted by all four examiners. The elliptical annuli drawn in green are the fits by examiner 1, whose values are listed in Tables 2 and 3, while the white, cyan, and yellow lines and arrowheads indicate the major and minor axes of the elliptical annuli fitted by the other three examiners. Figure $1 a$ shows a bubble where all four examiners agree rather well on the size and thickness of the bubble. There is some disagreement on orientation because the bubble is roughly circular. Figure $1 b$ shows a bubble where the dispersion in bubble size and thickness is a bit larger than the typical dispersion between the four examiners, but not as large as the most extreme dispersions. All four examiners found about the same orientation, but the bright emission sources in the left half of the image made it difficult to judge the full extent of the bubble. 
TABLE 2

Northern Bubble Parameters

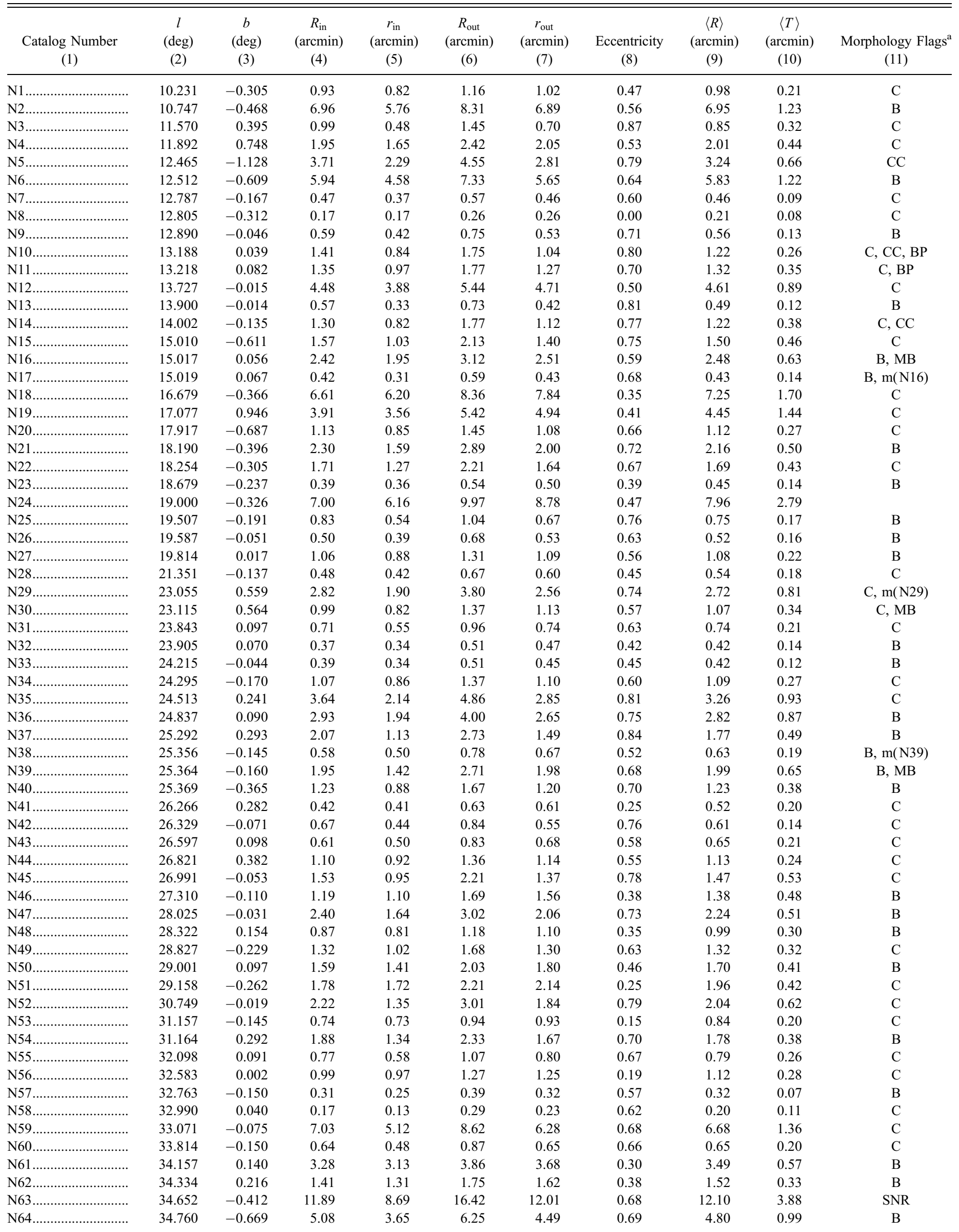


TABLE 2-Continued

\begin{tabular}{|c|c|c|c|c|c|c|c|c|c|c|}
\hline $\begin{array}{l}\text { Catalog Number } \\
\text { (1) }\end{array}$ & $\begin{array}{c}l \\
(\mathrm{deg}) \\
(2)\end{array}$ & $\begin{array}{c}b \\
(\mathrm{deg}) \\
(3)\end{array}$ & $\begin{array}{c}R_{\text {in }} \\
\text { (arcmin) } \\
(4)\end{array}$ & $\begin{array}{c}r_{\text {in }} \\
(\operatorname{arcmin}) \\
(5)\end{array}$ & $\begin{array}{c}R_{\text {out }} \\
\text { (arcmin) } \\
(6)\end{array}$ & $\begin{array}{c}r_{\text {out }} \\
\text { (arcmin) } \\
(7)\end{array}$ & $\begin{array}{l}\text { Eccentricity } \\
\text { (8) }\end{array}$ & $\begin{array}{c}\langle R\rangle \\
(\operatorname{arcmin}) \\
(9)\end{array}$ & $\begin{array}{c}\langle T\rangle \\
(\operatorname{arcmin}) \\
(10)\end{array}$ & $\begin{array}{c}\text { Morphology Flags }{ }^{\mathrm{a}} \\
\text { (11) }\end{array}$ \\
\hline 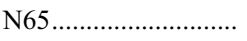 & 35.000 & 0.332 & 2.02 & 1.76 & 2.59 & 2.26 & 0.49 & 2.15 & 0.54 & $\mathrm{C}$ \\
\hline 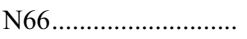 & 35.259 & 0.119 & 0.46 & 0.37 & 0.60 & 0.47 & 0.62 & 0.47 & 0.12 & $\mathrm{C}$ \\
\hline 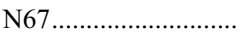 & 35.543 & 0.011 & 0.75 & 0.69 & 1.02 & 0.94 & 0.38 & 0.85 & 0.26 & $\mathrm{C}$ \\
\hline 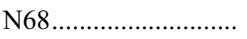 & 35.654 & -0.062 & 5.17 & 3.59 & 6.06 & 4.21 & 0.72 & 4.68 & 0.74 & $\mathrm{C}, \mathrm{CC}$ \\
\hline 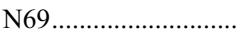 & 36.187 & 0.648 & 8.42 & 8.42 & 10.18 & 10.18 & 0.00 & 9.30 & 1.76 & $\mathrm{~B}$ \\
\hline 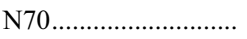 & 37.751 & -0.112 & 0.50 & 0.44 & 0.71 & 0.63 & 0.46 & 0.57 & 0.20 & $\mathrm{C}$ \\
\hline 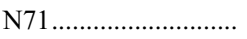 & 38.290 & 0.007 & 7.08 & 6.01 & 9.36 & 7.95 & 0.53 & 7.57 & 2.10 & $\mathrm{~B}$ \\
\hline 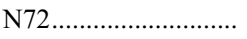 & 38.352 & -0.133 & 0.92 & 0.86 & 1.14 & 1.06 & 0.36 & 1.00 & 0.21 & B \\
\hline N73 ............................ & 38.739 & -0.137 & 0.77 & 0.72 & 1.15 & 1.08 & 0.34 & 0.93 & 0.37 & B \\
\hline 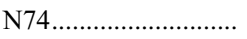 & 38.909 & -0.437 & 1.27 & 1.02 & 1.79 & 1.44 & 0.59 & 1.37 & 0.47 & $\mathrm{C}, \mathrm{CC}$ \\
\hline N75 & 38.928 & -0.387 & 0.62 & 0.51 & 0.93 & 0.77 & 0.57 & 0.70 & 0.29 & $\mathrm{~B}$ \\
\hline N76............................ & 38.955 & -0.731 & 3.73 & 2.84 & 5.27 & 4.02 & 0.65 & 3.93 & 1.34 & B \\
\hline 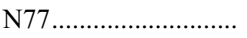 & 40.421 & -0.056 & 1.16 & 1.10 & 1.49 & 1.41 & 0.33 & 1.29 & 0.32 & B \\
\hline 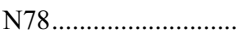 & 41.229 & 0.170 & 0.31 & 0.23 & 0.45 & 0.33 & 0.68 & 0.33 & 0.12 & $\mathrm{C}$ \\
\hline 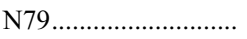 & 41.514 & 0.030 & 1.27 & 0.98 & 1.60 & 1.23 & 0.64 & 1.26 & 0.29 & $\mathrm{C}$ \\
\hline ................... & 41.930 & 0.031 & 1.90 & 1.38 & 2.38 & 1.73 & 0.69 & 1.82 & 0.41 & $\mathrm{C}$ \\
\hline 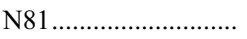 & 42.003 & -0.512 & 9.24 & 6.57 & 11.98 & 8.52 & 0.70 & 8.95 & 2.31 & $\mathrm{C}, \mathrm{MB}$ \\
\hline 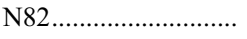 & 42.102 & -0.623 & 1.47 & 1.46 & 1.91 & 1.90 & 0.13 & 1.68 & 0.44 & $\mathrm{C}, \mathrm{CC}, \mathrm{m}(\mathrm{N} 81)$ \\
\hline 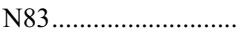 & 42.113 & -0.442 & 0.31 & 0.24 & 0.44 & 0.33 & 0.65 & 0.33 & 0.11 & $\mathrm{C}, \mathrm{m}(\mathrm{N} 81)$ \\
\hline 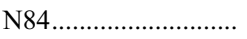 & 42.831 & -0.161 & 1.19 & 0.86 & 1.54 & 1.12 & 0.69 & 1.16 & 0.30 & $\mathrm{C}$ \\
\hline 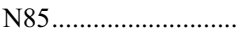 & 43.074 & -0.017 & 0.46 & 0.38 & 0.59 & 0.49 & 0.57 & 0.48 & 0.12 & $\mathrm{C}$ \\
\hline N86............................ & 43.097 & -0.040 & 0.26 & 0.23 & 0.43 & 0.38 & 0.46 & 0.32 & 0.17 & B \\
\hline 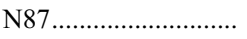 & 43.218 & 0.107 & 0.31 & 0.24 & 0.45 & 0.36 & 0.61 & 0.34 & 0.12 & $\mathrm{C}$ \\
\hline N88 ............................ & 43.265 & -0.186 & 1.37 & 1.30 & 1.90 & 1.81 & 0.30 & 1.59 & 0.52 & SNR \\
\hline 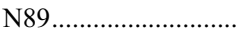 & 43.739 & 0.114 & 0.92 & 0.81 & 1.24 & 1.10 & 0.47 & 1.02 & 0.31 & $\mathrm{C}$ \\
\hline 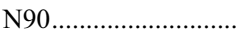 & 43.774 & 0.059 & 1.53 & 1.52 & 1.96 & 1.94 & 0.13 & 1.74 & 0.42 & $\mathrm{C}$ \\
\hline N91 ........................... & 44.211 & 0.050 & 5.19 & 3.47 & 7.25 & 4.84 & 0.74 & 5.08 & 1.68 & $\mathrm{C}$ \\
\hline 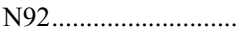 & 44.333 & -0.839 & 2.03 & 1.38 & 2.67 & 1.81 & 0.73 & 1.94 & 0.53 & $\mathrm{C}, \mathrm{CC}$ \\
\hline N93 ............................. & 44.777 & -0.550 & 0.73 & 0.53 & 1.00 & 0.73 & 0.68 & 0.74 & 0.23 & $\mathrm{C}, \mathrm{m}(\mathrm{N} 94)$ \\
\hline 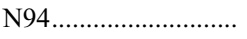 & 44.818 & -0.500 & 3.85 & 2.60 & 5.26 & 3.56 & 0.74 & 3.75 & 1.16 & $\mathrm{~B}, \mathrm{MB}$ \\
\hline 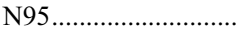 & 45.393 & -0.717 & 2.13 & 1.70 & 2.39 & 1.91 & 0.60 & 2.02 & 0.23 & $\mathrm{C}$ \\
\hline N96............................ & 46.947 & 0.368 & 0.40 & 0.33 & 0.53 & 0.43 & 0.59 & 0.42 & 0.12 & $\mathrm{~B}, \mathrm{MB}$ \\
\hline 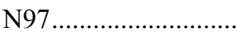 & 46.951 & 0.312 & 4.23 & 3.13 & 5.59 & 4.14 & 0.67 & 4.23 & 1.17 & $\mathrm{~B}, \mathrm{~m}(\mathrm{~N} 96)$ \\
\hline 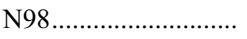 & 47.027 & 0.219 & 1.37 & 1.36 & 1.75 & 1.74 & 0.11 & 1.56 & 0.38 & $\mathrm{C}$ \\
\hline 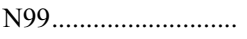 & 48.540 & 0.468 & 4.20 & 3.23 & 5.74 & 4.41 & 0.64 & 4.36 & 1.35 & B \\
\hline 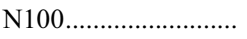 & 48.821 & -0.557 & 4.85 & 3.78 & 6.23 & 4.86 & 0.63 & 4.89 & 1.22 & $\mathrm{C}$ \\
\hline 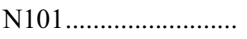 & 49.197 & -0.358 & 0.99 & 0.85 & 1.53 & 1.31 & 0.52 & 1.17 & 0.50 & $\mathrm{~B}$ \\
\hline N102 .......................... & 49.698 & -0.162 & 1.82 & 1.81 & 2.20 & 2.18 & 0.12 & 2.00 & 0.38 & $\mathrm{C}, \mathrm{MB}$ \\
\hline 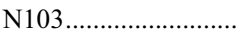 & 49.703 & -0.168 & 0.63 & 0.49 & 0.87 & 0.68 & 0.63 & 0.67 & 0.21 & $\mathrm{~B}, \mathrm{~m}(\mathrm{~N} 102)$ \\
\hline N104......................... & 50.041 & -0.273 & 0.77 & 0.59 & 1.09 & 0.84 & 0.64 & 0.81 & 0.29 & $\mathrm{C}$ \\
\hline N105 „......................... & 50.077 & 0.569 & 0.87 & 0.57 & 1.19 & 0.79 & 0.75 & 0.84 & 0.26 & $\mathrm{C}$ \\
\hline 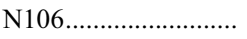 & 50.785 & 0.168 & 0.39 & 0.30 & 0.56 & 0.43 & 0.65 & 0.41 & 0.15 & $\mathrm{C}, \mathrm{m}(\mathrm{N} 107)$ \\
\hline N107 ........................... & 50.972 & 0.078 & 11.59 & 9.08 & 14.14 & 11.08 & 0.62 & 11.39 & 2.26 & $\mathrm{~B}, \mathrm{MB}$ \\
\hline 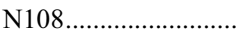 & 51.766 & 0.471 & 4.39 & 2.39 & 6.35 & 3.46 & 0.84 & 3.96 & 1.45 & $\mathrm{C}, \mathrm{m}(\mathrm{N} 109)$ \\
\hline N109......................... & 51.982 & 0.562 & 14.57 & 11.59 & 18.67 & 14.86 & 0.61 & 14.83 & 3.66 & $\mathrm{~B}, \mathrm{MB}$ \\
\hline 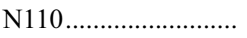 & 52.159 & 0.710 & 0.55 & 0.51 & 0.76 & 0.70 & 0.38 & 0.63 & 0.20 & $\mathrm{C}, \mathrm{m}(\mathrm{N} 109)$ \\
\hline N111 .......................... & 52.204 & 0.750 & 1.00 & 0.81 & 1.36 & 1.10 & 0.59 & 1.06 & 0.33 & $\mathrm{~B}, \mathrm{~m}(\mathrm{~N} 109)$ \\
\hline N112 ........................ & 52.206 & 0.721 & 0.28 & 0.19 & 0.38 & 0.26 & 0.74 & 0.27 & 0.08 & $\mathrm{C}, \mathrm{m}(\mathrm{N} 109)$ \\
\hline 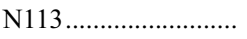 & 52.229 & 0.737 & 0.57 & 0.34 & 0.89 & 0.54 & 0.80 & 0.57 & 0.25 & $\mathrm{C}, \mathrm{CC}, \mathrm{m}(\mathrm{N} 109)$ \\
\hline N114 ......................... & 52.250 & 0.703 & 1.35 & 1.32 & 1.82 & 1.78 & 0.20 & 1.57 & 0.47 & $\mathrm{C}, \mathrm{m}(\mathrm{N} 109)$ \\
\hline N115 ......................... & 53.556 & -0.014 & 3.20 & 2.52 & 3.96 & 3.11 & 0.62 & 3.17 & 0.67 & $\mathrm{C}$ \\
\hline N116 ......................... & 54.091 & -0.088 & 0.79 & 0.63 & 1.05 & 0.83 & 0.61 & 0.82 & 0.23 & $\mathrm{~B}, \mathrm{BP}$ \\
\hline N117 ......................... & 54.112 & -0.064 & 1.41 & 1.40 & 1.92 & 1.90 & 0.14 & 1.66 & 0.50 & $\mathrm{C}, \mathrm{BP}$ \\
\hline N118 .......................... & 54.928 & -0.477 & 0.32 & 0.26 & 0.54 & 0.44 & 0.58 & 0.39 & 0.20 & $\mathrm{C}$ \\
\hline N119 ........................ & 55.157 & 0.130 & 4.31 & 3.79 & 5.41 & 4.76 & 0.48 & 4.56 & 1.03 & $\mathrm{C}$ \\
\hline 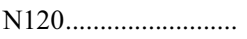 & 55.266 & 0.249 & 1.26 & 1.19 & 1.57 & 1.48 & 0.33 & 1.37 & 0.30 & $\mathrm{C}$ \\
\hline N121 _........................ & 55.445 & 0.886 & 0.49 & 0.41 & 0.69 & 0.58 & 0.55 & 0.54 & 0.19 & $\mathrm{C}$ \\
\hline N122 ........................ & 56.077 & -0.184 & 0.53 & 0.37 & 0.88 & 0.61 & 0.72 & 0.58 & 0.29 & $\mathrm{C}$ \\
\hline N123 ......................... & 57.539 & -0.284 & 1.47 & 1.01 & 1.85 & 1.27 & 0.73 & 1.38 & 0.31 & $\mathrm{C}$ \\
\hline N124........................ & 58.605 & 0.638 & 1.60 & 1.50 & 2.00 & 1.87 & 0.35 & 1.74 & 0.39 & B \\
\hline N125 ......................... & 58.764 & 0.655 & 0.74 & 0.67 & 0.95 & 0.85 & 0.44 & 0.80 & 0.20 & $\mathrm{C}$ \\
\hline 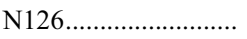 & 59.606 & 0.330 & 1.82 & 1.45 & 2.38 & 1.89 & 0.61 & 1.87 & 0.50 & $\mathrm{~B}, \mathrm{Fl}$ \\
\hline N127......................... & 60.648 & -0.057 & 2.94 & 2.88 & 3.80 & 3.72 & 0.20 & 3.34 & 0.85 & B \\
\hline N128......................... & 61.673 & 0.946 & 3.38 & 2.41 & 4.25 & 3.03 & 0.70 & 3.22 & 0.74 & B \\
\hline N129........................ & 61.755 & 0.839 & 3.27 & 2.34 & 4.14 & 2.96 & 0.70 & 3.13 & 0.73 & B \\
\hline
\end{tabular}


TABLE 2-Continued

\begin{tabular}{|c|c|c|c|c|c|c|c|c|c|c|}
\hline $\begin{array}{c}\text { Catalog Number } \\
\text { (1) }\end{array}$ & $\begin{array}{c}l \\
(\mathrm{deg}) \\
(2)\end{array}$ & $\begin{array}{c}b \\
(\mathrm{deg}) \\
(3)\end{array}$ & $\begin{array}{c}R_{\text {in }} \\
(\operatorname{arcmin}) \\
(4)\end{array}$ & $\begin{array}{c}r_{\text {in }} \\
(\operatorname{arcmin}) \\
(5)\end{array}$ & $\begin{array}{c}R_{\text {out }} \\
\text { (arcmin) } \\
(6)\end{array}$ & $\begin{array}{c}r_{\text {out }} \\
(\operatorname{arcmin}) \\
(7)\end{array}$ & $\begin{array}{c}\text { Eccentricity } \\
\text { (8) }\end{array}$ & $\begin{array}{c}\langle R\rangle \\
(\operatorname{arcmin}) \\
(9)\end{array}$ & $\begin{array}{c}\langle T\rangle \\
(\operatorname{arcmin}) \\
(10)\end{array}$ & $\begin{array}{c}\text { Morphology Flags } \\
\text { (11) }\end{array}$ \\
\hline N130.. & 62.370 & -0.540 & 0.98 & 0.87 & 1.26 & 1.11 & 0.47 & 1.05 & 0.26 & B \\
\hline …................. & 63.084 & -0.395 & 6.18 & 5.46 & 7.75 & 6.84 & 0.47 & 6.55 & 1.48 & $\mathrm{C}$ \\
\hline N132.. & 63.121 & 0.386 & 0.21 & 0.19 & 0.39 & 0.36 & 0.36 & 0.28 & 0.17 & $\mathrm{C}$ \\
\hline . & 63.159 & 0.451 & 1.83 & 1.14 & 2.33 & 1.45 & 0.78 & 1.64 & 0.39 & $\mathrm{C}$ \\
\hline N134...... & 64.134 & -0.466 & 0.58 & 0.32 & 0.92 & 0.51 & 0.83 & 0.56 & 0.25 & $\mathrm{~B}, \mathrm{CC}$ \\
\hline
\end{tabular}

Note.-Table 2 is also available in machine-readable form in the electronic edition of the Astrophysical Journal.

${ }^{a}$ The abbreviations in col. (11) are defined as follows: $\mathrm{C}$ is a complete or closed ring. B is a broken or incomplete ring. CC stands for a probable enclosed central

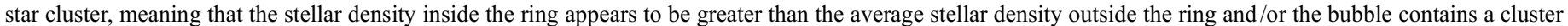
from a known catalog. MB stands for multiple bubbles, indicating a relatively large bubble having smaller secondary bubbles inside it or on its periphery. Subsidiary multiple bubbles are labeled m(catalog number) according to the multiple bubble they appear to be associated with. BP indicates a bipolar bubble or a double bubble whose lobes are in contact. TP indicates triple bubbles that appear to originate close to the same location. CS indicates that the central star(s) responsible for driving the bubble is/are probably apparent. Fl indicates a flocculent or clumpy bubble structure. The three bubbles that are identified as known supernova remnants are given the SNR flag.

Hence, Figure $1 b$ provides an example of the difficulties associated with measuring bubble geometrical parameters.

Every bubble in the catalog has been independently examined and verified by three of the authors. We believe that this screening process has provided a nearly $100 \%$ reliable catalog of bubbles. We caution, however, that identification of three-dimensional morphological features from two-dimensional images is subjective and prone to individual biases.

\subsection{The Catalog}

The detected bubbles and their measured parameters are listed in Tables 2 and 3 for the northern and southern regions, respectively. The catalog entries are in order of increasing angular distance from the Galactic center. The columns in Tables 2 and 3 are as follows: (1) the catalog number (preceded by $\mathrm{N}$ in Table 2, by $\mathrm{S}$ in Table 3; the S prefix does not refer to a Sharpless catalog number); (2) and (3) the center position in Galactic longitude and latitude, respectively; (4) and (5) the semimajor $\left(R_{\text {in }}\right)$ and semiminor $\left(r_{\text {in }}\right)$ axes of the inner ellipse, respectively; (6) and (7) the semimajor $\left(R_{\text {out }}\right)$ and semiminor $\left(r_{\text {out }}\right)$ axes of the outer ellipse, respectively; (8) the eccentricity of the ellipses (the centers and eccentricities of the inner and outer ellipses were constrained to be the same); (9) the average radius $\langle R\rangle$ of the bubble; (10) the average thickness $\langle T\rangle$ of the shell; and (11) abbreviated comments on morphological properties (see the footnote to Table 2 for a full description of the morphology flags). We estimate the average radius of each bubble as the average of the geometric mean radii of the inner and outer ellipses using

$$
\langle R\rangle=\frac{\left(R_{\text {out }} r_{\text {out }}\right)^{0.5}+\left(R_{\text {in }} r_{\text {in }}\right)^{0.5}}{2} .
$$

The average projected thickness is the difference between the geometric mean radii of the outer and inner ellipses and is calculated using

$$
\langle T\rangle=\left(R_{\text {out }} r_{\text {out }}\right)^{0.5}-\left(R_{\text {in }} r_{\text {in }}\right)^{0.5} .
$$

In Figures $2 a-2 h$ we show examples of bubbles that illustrate the morphological flags referred to in column (11) of Tables 2 and 3. We refer to individual bubbles by catalog number. All of the bubbles in these figures except S21 are located toward known $\mathrm{H}$ II regions, and all except S21 and S97+S98 probably contain star clusters previously detected in the near- to mid-IR ( $\operatorname{see} \S 3.1$ ). In Figure $2 a, \mathrm{~S} 133$ is a good representative of a bubble with smaller bubbles (S132, S134, and S135) within and on the rim of a larger bubble (multiple bubbles [MB]). In Figure $2 b, \mathrm{RCW} 79$ (S145) is an example of a broken bubble (B) with a secondary small bubble (S144) on its rim (MB). A compact star cluster is also located within the large bubble (CC). In Figure $2 c$, S44 is an example of a complete (C) bubble. In Figure $2 d$, S21 is an example of a complete (C) bubble with a bright central star (CS) that may be responsible for producing the bubble. In Figure $2 e, \mathrm{~S} 156$ has a central star cluster (CC) within the bubble. The bubble exhibits a predominantly filamentary structure. In Figure $2 f$, S97+ S98 together form a double or bipolar bubble (BP) with a cluster of stars (CC) along the line of contact between the two bubble lobes. In Figure $2 g, \mathrm{~S} 109, \mathrm{~S} 110$, and S111 form an example of a triple or tripolar bubble (TP). Finally, S100, in Figure $2 h$, is a good example of an irregular bubble exhibiting flocculent $(\mathrm{Fl})$ structure.

We caution that perceived morphological properties are observer and wavelength dependent. We have attempted to fit a wide range of bubble morphologies using elliptical annuli. In some cases an elliptical annulus is not a good representation of the morphology. The morphological designations given in column (11) of Tables 2 and 3 are abbreviated descriptions of complicated structures. We strongly recommend that the online image archive (see footnote 13) be examined for particular bubbles of interest.

\section{RESULTS AND DISCUSSION}

In the following, we use the measured parameters tabulated in Tables 2 and 3 to investigate statistical properties of the bubbles in the inner Galaxy. In particular, we examine what types of objects produce the observed bubbles, the distribution of the bubbles in longitude and latitude, and the distributions of bubble size, thickness, and eccentricity. The motivations for this are to show the frequency and range of bubble properties in the GLIMPSE sample and to provide constraints needed to model the process of bubble formation.

\subsection{Wavelength Dependence}

The bubbles found in GLIMPSE are generally detectable in all four IRAC bands but tend to be brighter at longer wavelengths. Most bubbles are larger and brighter at $8.0 \mu \mathrm{m}$ than the shorter wavelength IRAC bands. In Figure 3 we show color images of bubbles (a) N107 and (b) N100-N113, in the $4.5 \mu \mathrm{m}$ (blue) and $8.0 \mu \mathrm{m}$ (green) bands from GLIMPSE and the $24 \mu \mathrm{m}($ red $)$ band from the MIPSGAL (S. Carey, PI) survey. In this image, the $24 \mu \mathrm{m}$ emission dominates inside of the bubbles and often, but not always, seems to be confined to regions close to hot or bright 
TABLE 3

Southern Bubble Parameters

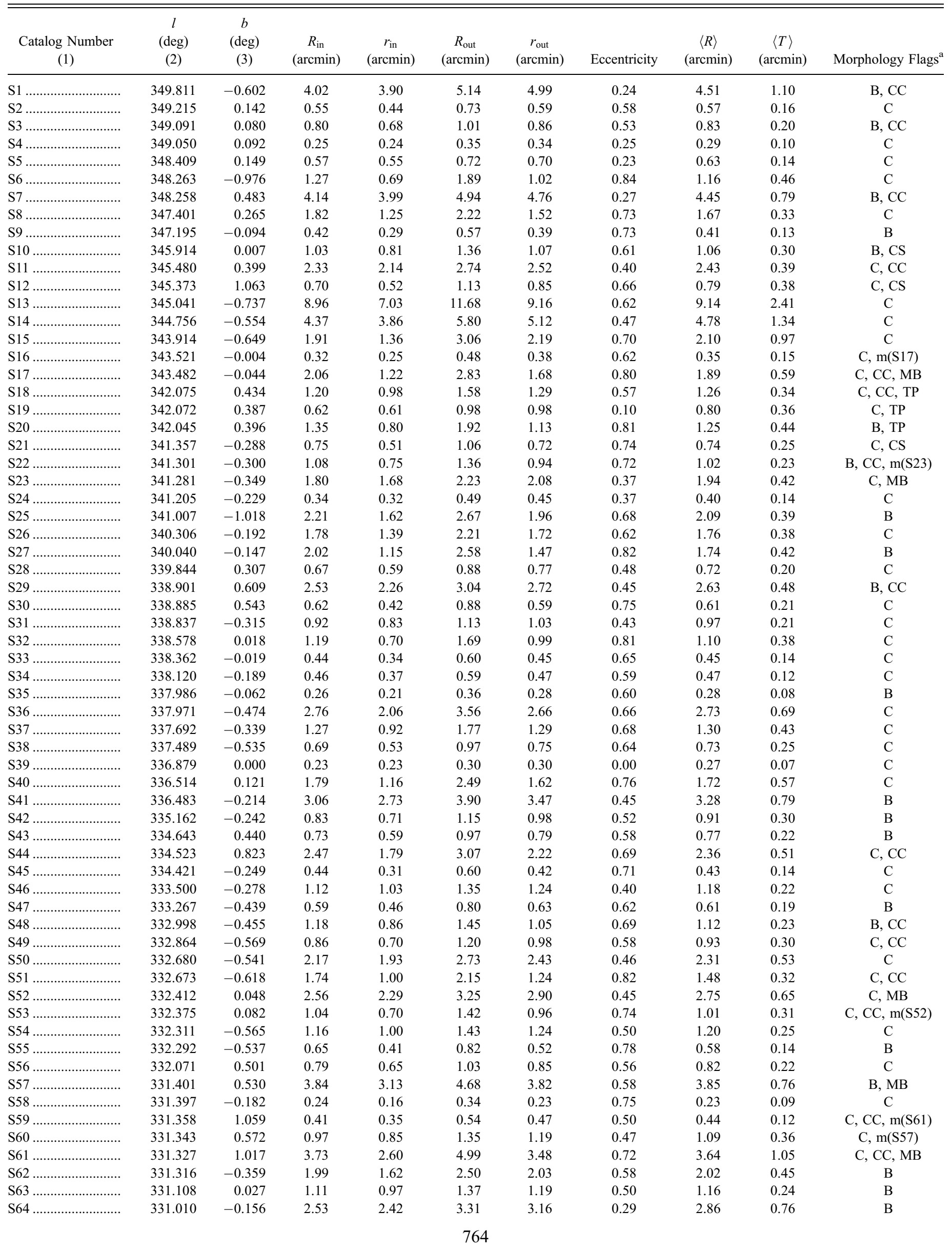


TABLE 3-Continued

\begin{tabular}{|c|c|c|c|c|c|c|c|c|c|c|}
\hline $\begin{array}{c}\text { Catalog Number } \\
\text { (1) }\end{array}$ & $\begin{array}{c}l \\
(\mathrm{deg}) \\
(2)\end{array}$ & $\begin{array}{c}b \\
(\mathrm{deg}) \\
(3)\end{array}$ & $\begin{array}{c}R_{\text {in }} \\
(\operatorname{arcmin})\end{array}$ & $\begin{array}{c}r_{\text {in }} \\
(\operatorname{arcmin})\end{array}$ & $\begin{array}{c}R_{\text {out }} \\
\text { (arcmin) }\end{array}$ & $\begin{array}{c}r_{\text {out }} \\
(\operatorname{arcmin})\end{array}$ & Eccentricity & $\begin{array}{c}\langle R\rangle \\
(\operatorname{arcmin})\end{array}$ & $\begin{array}{c}\langle T\rangle \\
(\operatorname{arcmin})\end{array}$ & Morphology Flags $^{\mathrm{a}}$ \\
\hline 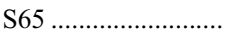 & 330.789 & -0.454 & 0.56 & 0.34 & 0.75 & 0.45 & 0.80 & 0.51 & 0.14 & $\mathrm{C}, \mathrm{m}(\mathrm{S} 66)$ \\
\hline S66 ............................. & 330.781 & -0.414 & 5.81 & 4.20 & 7.42 & 5.37 & 0.69 & 5.63 & 1.37 & $\mathrm{~B}, \mathrm{CC}, \mathrm{MB}$ \\
\hline 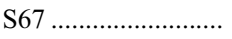 & 330.675 & -0.389 & 0.99 & 0.65 & 1.38 & 0.91 & 0.76 & 0.96 & 0.32 & $\mathrm{C}, \mathrm{CC}, \mathrm{m}(\mathrm{S} 66)$ \\
\hline 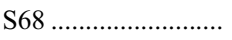 & 329.975 & -0.003 & 1.02 & 0.74 & 1.45 & 1.05 & 0.69 & 1.05 & 0.36 & B \\
\hline S69 …......................... & 329.657 & -0.486 & 1.07 & 1.06 & 1.48 & 1.46 & 0.15 & 1.27 & 0.41 & $\mathrm{C}, \mathrm{CC}$ \\
\hline 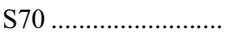 & 329.276 & 0.108 & 1.04 & 0.81 & 1.39 & 1.08 & 0.63 & 1.07 & 0.31 & $\mathrm{~B}$ \\
\hline 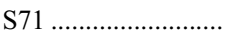 & 327.990 & -0.103 & 1.03 & 0.82 & 1.37 & 1.09 & 0.60 & 1.07 & 0.30 & $\mathrm{C}, \mathrm{CC}$ \\
\hline S72 & 327.705 & 0.567 & 1.73 & 1.10 & 2.31 & 1.48 & 0.77 & 1.61 & 0.47 & $\mathrm{~B}$ \\
\hline 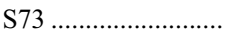 & 327.552 & -0.807 & 3.71 & 2.26 & 5.16 & 3.14 & 0.79 & 3.46 & 1.13 & $\mathrm{~B}, \mathrm{CC}, \mathrm{MB}$ \\
\hline 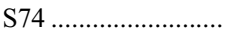 & 327.525 & -0.848 & 1.37 & 0.98 & 1.78 & 1.28 & 0.70 & 1.33 & 0.35 & $\mathrm{C}, \mathrm{m}(\mathrm{S} 73)$ \\
\hline S75 …...................... & 327.141 & -0.264 & 0.43 & 0.41 & 0.59 & 0.56 & 0.28 & 0.50 & 0.15 & $\mathrm{C}$ \\
\hline 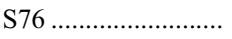 & 326.924 & -0.021 & 4.55 & 4.16 & 5.77 & 5.28 & 0.40 & 4.94 & 1.17 & $\mathrm{~B}$ \\
\hline 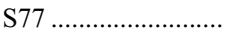 & 326.918 & -1.098 & 1.24 & 0.81 & 1.68 & 1.09 & 0.76 & 1.18 & 0.36 & $\mathrm{~B}, \mathrm{CC}$ \\
\hline 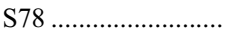 & 326.783 & -0.551 & 2.82 & 1.98 & 3.44 & 2.41 & 0.71 & 2.62 & 0.52 & $\mathrm{C}$ \\
\hline S79 & 326.700 & 0.513 & 2.69 & 1.84 & 3.45 & 2.36 & 0.73 & 2.54 & 0.63 & $\mathrm{C}, \mathrm{CC}$ \\
\hline 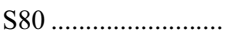 & 325.014 & -0.137 & 5.81 & 4.30 & 7.59 & 5.62 & 0.67 & 5.76 & 1.53 & $\mathrm{C}$ \\
\hline S81 ........................... & 324.642 & -0.311 & 1.20 & 0.88 & 1.58 & 1.17 & 0.67 & 1.19 & 0.33 & $\mathrm{~B}$ \\
\hline S82 …...................... & 324.057 & -0.813 & 4.41 & 3.79 & 5.94 & 5.10 & 0.51 & 4.80 & 1.42 & $\mathrm{C}$ \\
\hline S83 …...................... & 323.975 & 0.057 & 0.42 & 0.34 & 0.56 & 0.45 & 0.59 & 0.44 & 0.13 & $\mathrm{C}$ \\
\hline S84 „........................... & 323.915 & 0.032 & 0.33 & 0.23 & 0.50 & 0.35 & 0.70 & 0.35 & 0.14 & $\mathrm{C}$ \\
\hline 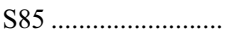 & 323.802 & 0.025 & 1.48 & 1.26 & 1.93 & 1.65 & 0.52 & 1.58 & 0.42 & $\mathrm{~B}, \mathrm{CC}$ \\
\hline S86 …........................ & 323.538 & -0.352 & 0.82 & 0.79 & 1.21 & 1.17 & 0.26 & 1.00 & 0.39 & $\mathrm{C}$ \\
\hline 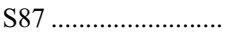 & 322.418 & 0.207 & 0.30 & 0.15 & 0.44 & 0.23 & 0.85 & 0.26 & 0.10 & $\mathrm{C}$ \\
\hline 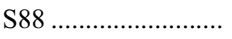 & 321.672 & -0.041 & 0.46 & 0.41 & 0.63 & 0.56 & 0.46 & 0.51 & 0.16 & $\mathrm{~B}$ \\
\hline 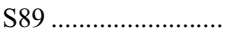 & 321.394 & -0.317 & 0.90 & 0.70 & 1.16 & 0.90 & 0.63 & 0.91 & 0.23 & $\mathrm{C}, \mathrm{CC}$ \\
\hline 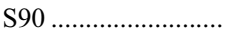 & 320.947 & -0.417 & 0.30 & 0.24 & 0.46 & 0.36 & 0.62 & 0.34 & 0.14 & $\mathrm{~B}$ \\
\hline S91 …....................... & 320.861 & -0.422 & 2.31 & 1.77 & 2.91 & 2.23 & 0.64 & 2.29 & 0.53 & B \\
\hline S92 …........................... & 320.578 & 0.203 & 6.18 & 5.08 & 7.90 & 6.49 & 0.57 & 6.38 & 1.55 & B \\
\hline S93 …....................... & 320.438 & 0.251 & 2.47 & 1.94 & 3.18 & 2.50 & 0.62 & 2.50 & 0.63 & $\mathrm{C}$ \\
\hline S94 „......................... & 320.227 & -0.110 & 2.01 & 1.31 & 2.43 & 1.58 & 0.76 & 1.79 & 0.34 & $\mathrm{~B}$ \\
\hline S95 …....................... & 320.205 & -0.346 & 0.49 & 0.42 & 0.72 & 0.62 & 0.50 & 0.56 & 0.21 & $\mathrm{~B}$ \\
\hline 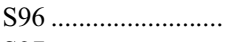 & 320.164 & 0.793 & 1.35 & 1.11 & 1.80 & 1.49 & 0.56 & 1.43 & 0.41 & $\mathrm{C}$ \\
\hline S97 .......................... & 319.890 & 0.763 & 1.51 & 1.05 & 2.14 & 1.50 & 0.72 & 1.52 & 0.53 & $\mathrm{~B}, \mathrm{CC}, \mathrm{BP}$ \\
\hline S98 …....................... & 319.884 & 0.808 & 0.82 & 0.62 & 1.28 & 0.97 & 0.65 & 0.91 & 0.40 & $\mathrm{C}, \mathrm{CC}, \mathrm{BP}$ \\
\hline 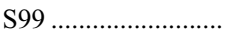 & 319.224 & 0.229 & 2.32 & 1.71 & 3.00 & 2.21 & 0.68 & 2.28 & 0.58 & $\mathrm{~B}$ \\
\hline $\mathrm{S} 100 \ldots \ldots \ldots \ldots \ldots \ldots \ldots \ldots$ & 319.145 & -0.345 & 3.52 & 2.50 & 4.75 & 3.37 & 0.70 & 3.48 & 1.04 & $\mathrm{C}, \mathrm{CC}, \mathrm{MB}$ \\
\hline 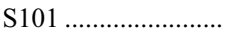 & 319.135 & -0.413 & 0.62 & 0.49 & 0.88 & 0.70 & 0.61 & 0.67 & 0.24 & $\mathrm{C}, \mathrm{m}(\mathrm{S} 100)$ \\
\hline 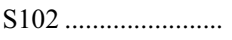 & 318.064 & -0.083 & 0.24 & 0.19 & 0.38 & 0.30 & 0.62 & 0.27 & 0.12 & $\mathrm{C}$ \\
\hline S103 „........................ & 317.997 & -0.611 & 3.21 & 2.82 & 4.49 & 3.95 & 0.48 & 3.61 & 1.20 & $\mathrm{C}$ \\
\hline 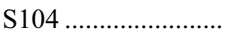 & 317.988 & -0.749 & 1.71 & 1.13 & 2.55 & 1.68 & 0.75 & 1.73 & 0.68 & $\mathrm{C}$ \\
\hline 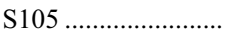 & 317.798 & -0.770 & 0.25 & 0.21 & 0.37 & 0.31 & 0.56 & 0.28 & 0.11 & $\mathrm{~B}$ \\
\hline S106 ......................... & 317.462 & -0.347 & 1.68 & 1.65 & 2.02 & 1.98 & 0.19 & 1.83 & 0.34 & $\mathrm{~B}, \mathrm{CC}$ \\
\hline S107 ........................ & 317.212 & 0.103 & 0.25 & 0.21 & 0.42 & 0.35 & 0.57 & 0.31 & 0.15 & $\mathrm{~B}$ \\
\hline S108 …....................... & 316.866 & 0.030 & 2.84 & 2.59 & 4.04 & 3.68 & 0.41 & 3.28 & 1.14 & $\mathrm{C}$ \\
\hline S109 ......................... & 316.812 & -0.101 & 1.51 & 0.96 & 2.32 & 1.47 & 0.77 & 1.52 & 0.64 & $\mathrm{C}, \mathrm{TP}$ \\
\hline 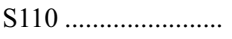 & 316.809 & -0.031 & 1.41 & 1.09 & 1.82 & 1.41 & 0.63 & 1.42 & 0.36 & $\mathrm{C}, \mathrm{TP}$ \\
\hline S111_........................ & 316.772 & -0.068 & 1.37 & 0.98 & 1.83 & 1.31 & 0.70 & 1.35 & 0.39 & $\mathrm{C}, \mathrm{CC}, \mathrm{TP}$ \\
\hline S112 ….................... & 316.671 & 0.370 & 0.24 & 0.17 & 0.42 & 0.29 & 0.72 & 0.27 & 0.15 & $\mathrm{C}$ \\
\hline 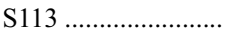 & 316.544 & 0.004 & 1.41 & 1.15 & 1.98 & 1.61 & 0.58 & 1.53 & 0.51 & B \\
\hline 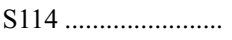 & 316.526 & -0.601 & 1.28 & 1.09 & 1.81 & 1.54 & 0.52 & 1.43 & 0.49 & $\mathrm{~B}, \mathrm{CC}$ \\
\hline S115 „........................ & 315.979 & -0.182 & 0.83 & 0.83 & 1.06 & 1.06 & 0.00 & 0.94 & 0.23 & $\mathrm{C}, \mathrm{CC}$ \\
\hline S116 „......................... & 314.219 & 0.474 & 5.10 & 3.00 & 5.81 & 3.41 & 0.81 & 4.18 & 0.54 & $\mathrm{C}, \mathrm{CC}$ \\
\hline 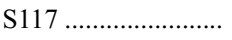 & 314.215 & 0.340 & 1.04 & 0.69 & 1.50 & 1.00 & 0.74 & 1.04 & 0.38 & $\mathrm{C}, \mathrm{CC}$ \\
\hline $\mathrm{S} 118 \ldots \ldots \ldots \ldots \ldots \ldots \ldots$ & 314.186 & 0.275 & 2.12 & 1.72 & 2.58 & 2.09 & 0.59 & 2.12 & 0.41 & $\mathrm{~B}$ \\
\hline S119 ........................ & 313.976 & 0.136 & 0.61 & 0.58 & 0.77 & 0.74 & 0.29 & 0.67 & 0.16 & $\mathrm{~B}, \mathrm{CS}$ \\
\hline S120 ......................... & 313.810 & 0.133 & 2.01 & 1.62 & 2.49 & 2.00 & 0.59 & 2.02 & 0.42 & $\mathrm{C}$ \\
\hline S121 ........................ & 313.792 & 0.701 & 1.14 & 0.85 & 1.52 & 1.14 & 0.67 & 1.15 & 0.33 & $\mathrm{C}, \mathrm{CC}$ \\
\hline 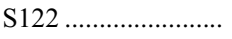 & 313.418 & 0.128 & 7.03 & 4.41 & 8.69 & 5.45 & 0.78 & 6.22 & 1.31 & $\mathrm{C}$ \\
\hline S123 „........................ & 312.975 & -0.433 & 2.16 & 1.94 & 2.75 & 2.46 & 0.45 & 2.32 & 0.55 & $\mathrm{C}, \mathrm{CC}$ \\
\hline S124 „....................... & 312.888 & -0.440 & 0.81 & 0.45 & 1.38 & 0.77 & 0.83 & 0.82 & 0.43 & $\mathrm{C}$ \\
\hline S125 ......................... & 312.760 & 0.072 & 0.77 & 0.51 & 1.02 & 0.68 & 0.75 & 0.73 & 0.21 & $\mathrm{~B}$ \\
\hline S126 ........................ & 312.705 & 0.015 & 1.04 & 0.97 & 1.50 & 1.40 & 0.36 & 1.23 & 0.45 & $\mathrm{~B}, \mathrm{CC}$ \\
\hline S127 ........................ & 312.698 & -0.115 & 1.90 & 1.42 & 2.38 & 1.78 & 0.67 & 1.85 & 0.41 & $\mathrm{~B}, \mathrm{BP}$ \\
\hline S128 ......................... & 312.675 & 0.047 & 1.40 & 1.28 & 1.78 & 1.63 & 0.40 & 1.52 & 0.37 & $\mathrm{C}, \mathrm{CC}, \mathrm{BP}$ \\
\hline S129 ......................... & 312.537 & 0.061 & 0.32 & 0.18 & 0.50 & 0.28 & 0.83 & 0.30 & 0.13 & $\mathrm{~B}$ \\
\hline
\end{tabular}


TABLE 3-Continued

\begin{tabular}{|c|c|c|c|c|c|c|c|c|c|c|}
\hline $\begin{array}{c}\text { Catalog Number } \\
\text { (1) }\end{array}$ & $\begin{array}{c}l \\
(\mathrm{deg}) \\
(2)\end{array}$ & $\begin{array}{c}b \\
(\mathrm{deg}) \\
(3)\end{array}$ & $\begin{array}{c}R_{\text {in }} \\
(\operatorname{arcmin})\end{array}$ & $\begin{array}{c}r_{\text {in }} \\
(\operatorname{arcmin})\end{array}$ & $\begin{array}{c}R_{\text {out }} \\
(\operatorname{arcmin})\end{array}$ & $\begin{array}{c}r_{\text {out }} \\
(\operatorname{arcmin})\end{array}$ & Eccentricity & $\begin{array}{c}\langle R\rangle \\
(\operatorname{arcmin})\end{array}$ & $\begin{array}{c}\langle T\rangle \\
(\operatorname{arcmin})\end{array}$ & Morphology Flags \\
\hline $\mathrm{S} 130 \ldots \ldots \ldots \ldots \ldots \ldots \ldots$ & 312.151 & -0.313 & 0.40 & 0.34 & 0.57 & 0.49 & 0.51 & 0.45 & 0.16 & $\mathrm{~B}$ \\
\hline 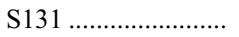 & 311.524 & -0.339 & 1.37 & 0.94 & 1.87 & 1.28 & 0.73 & 1.34 & 0.41 & SNR \\
\hline $\mathrm{S} 132 \ldots \ldots \ldots \ldots \ldots \ldots \ldots$ & 311.495 & 0.416 & 0.13 & 0.13 & 0.24 & 0.23 & 0.27 & 0.18 & 0.10 & $\mathrm{C}, \mathrm{m}(\mathrm{S} 133)$ \\
\hline S133 ....................... & 311.487 & 0.395 & 1.86 & 1.64 & 2.45 & 2.17 & 0.47 & 2.03 & 0.56 & $\mathrm{C}, \mathrm{MB}$ \\
\hline $\mathrm{S} 134 \ldots \ldots \ldots \ldots \ldots \ldots \ldots \ldots$ & 311.484 & 0.363 & 0.57 & 0.47 & 0.74 & 0.61 & 0.55 & 0.60 & 0.15 & $\mathrm{~B}, \mathrm{CC}, \mathrm{m}(\mathrm{S} 133)$ \\
\hline 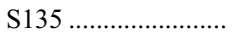 & 311.474 & 0.403 & 0.33 & 0.24 & 0.46 & 0.33 & 0.70 & 0.34 & 0.11 & $\mathrm{~B}, \mathrm{~m}(\mathrm{~S} 133)$ \\
\hline S136 ..................... & 311.189 & 0.751 & 0.66 & 0.42 & 0.89 & 0.57 & 0.77 & 0.62 & 0.19 & $\mathrm{C}$ \\
\hline $\mathrm{S} 137 \ldots \ldots$. & 310.980 & 0.411 & 2.98 & 2.38 & 4.00 & 3.20 & 0.60 & 3.12 & 0.91 & $\mathrm{C}, \mathrm{CC}$ \\
\hline $\mathrm{S} 138 \ldots \ldots \ldots \ldots \ldots \ldots \ldots \ldots$ & 310.909 & -0.361 & 0.78 & 0.72 & 1.29 & 1.18 & 0.40 & 0.99 & 0.49 & B \\
\hline 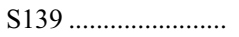 & 310.887 & 0.011 & 0.35 & 0.29 & 0.45 & 0.38 & 0.53 & 0.37 & 0.10 & $\mathrm{C}$ \\
\hline $\mathrm{S} 140 \ldots \ldots \ldots \ldots \ldots \ldots \ldots$ & 310.666 & -0.449 & 0.88 & 0.72 & 1.13 & 0.92 & 0.58 & 0.91 & 0.22 & $\mathrm{~B}$ \\
\hline S141 ........................ & 309.552 & -0.719 & 1.48 & 1.13 & 1.86 & 1.42 & 0.65 & 1.46 & 0.33 & $\mathrm{C}, \mathrm{Fl}$ \\
\hline S142 „...................... & 309.410 & -0.369 & 1.83 & 1.39 & 2.29 & 1.74 & 0.65 & 1.80 & 0.40 & $\mathrm{~B}$ \\
\hline S143 ...................... & 309.052 & 0.172 & 5.09 & 3.57 & 6.08 & 4.27 & 0.71 & 4.68 & 0.83 & $\mathrm{C}, \mathrm{CC}$ \\
\hline 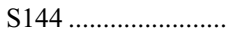 & 308.749 & 0.549 & 0.79 & 0.74 & 0.99 & 0.93 & 0.34 & 0.86 & 0.19 & $\mathrm{~B}, \mathrm{CC}, \mathrm{m}(\mathrm{S} 145)$ \\
\hline 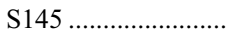 & 308.709 & 0.627 & 5.77 & 4.78 & 6.93 & 5.73 & 0.56 & 5.78 & 1.05 & $\mathrm{~B}, \mathrm{CC}, \mathrm{MB}$ \\
\hline 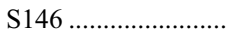 & 307.795 & -0.483 & 0.32 & 0.24 & 0.47 & 0.35 & 0.66 & 0.34 & 0.13 & $\mathrm{C}$ \\
\hline 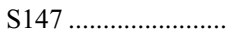 & 306.745 & 0.263 & 1.95 & 1.48 & 2.63 & 1.99 & 0.65 & 1.99 & 0.59 & $\mathrm{C}$ \\
\hline 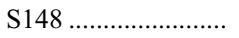 & 305.640 & 0.511 & 0.56 & 0.53 & 0.74 & 0.70 & 0.34 & 0.63 & 0.17 & $\mathrm{C}$ \\
\hline S149 „....................... & 305.553 & 0.010 & 0.37 & 0.33 & 0.58 & 0.51 & 0.48 & 0.45 & 0.20 & $\mathrm{~B}, \mathrm{CC}$ \\
\hline $\mathrm{S} 150 \ldots \ldots \ldots \ldots \ldots \ldots \ldots \ldots \ldots$ & 305.533 & 0.357 & 1.08 & 0.76 & 1.43 & 1.01 & 0.71 & 1.06 & 0.29 & $\mathrm{C}, \mathrm{CC}$ \\
\hline 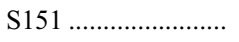 & 305.438 & -0.230 & 0.26 & 0.20 & 0.44 & 0.33 & 0.66 & 0.31 & 0.15 & $\mathrm{C}$ \\
\hline $\mathrm{S} 152 \ldots \ldots \ldots \ldots \ldots \ldots \ldots \ldots$ & 305.385 & -0.244 & 1.39 & 1.00 & 1.71 & 1.23 & 0.69 & 1.31 & 0.27 & $\mathrm{C}, \mathrm{CC}, \mathrm{Fl}$ \\
\hline S153 ........................ & 305.353 & 0.197 & 0.29 & 0.17 & 0.46 & 0.26 & 0.82 & 0.28 & 0.13 & $\mathrm{C}$ \\
\hline 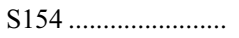 & 305.316 & 0.063 & 0.37 & 0.29 & 0.60 & 0.47 & 0.63 & 0.43 & 0.21 & $\mathrm{~B}$ \\
\hline 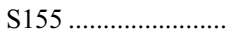 & 305.263 & -0.002 & 0.38 & 0.36 & 0.58 & 0.55 & 0.33 & 0.47 & 0.19 & $\mathrm{C}, \mathrm{CC}$ \\
\hline S156 ......................... & 305.263 & 0.214 & 1.63 & 1.62 & 2.16 & 2.15 & 0.13 & 1.89 & 0.53 & $\mathrm{C}, \mathrm{CC}$ \\
\hline S157 ..................... & 305.140 & -0.134 & 1.06 & 1.05 & 1.36 & 1.35 & 0.11 & 1.21 & 0.30 & $\mathrm{C}$ \\
\hline S158 ........................ & 304.660 & -0.857 & 6.56 & 6.09 & 8.76 & 8.13 & 0.37 & 7.38 & 2.12 & $\mathrm{C}, \mathrm{MB}$ \\
\hline 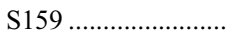 & 304.545 & -0.841 & 0.31 & 0.30 & 0.50 & 0.48 & 0.33 & 0.40 & 0.19 & $\mathrm{C}, \mathrm{m}(\mathrm{S} 158)$ \\
\hline 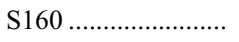 & 304.464 & -0.018 & 0.60 & 0.60 & 0.81 & 0.81 & 0.00 & 0.71 & 0.22 & $\mathrm{~B}, \mathrm{BP}$ \\
\hline S161 ....................... & 304.445 & -0.023 & 0.55 & 0.37 & 0.94 & 0.63 & 0.74 & 0.61 & 0.32 & $\mathrm{C}, \mathrm{BP}$ \\
\hline 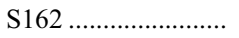 & 304.021 & 0.448 & 0.94 & 0.68 & 1.31 & 0.95 & 0.69 & 0.96 & 0.32 & $\mathrm{C}$ \\
\hline S163 ........................ & 303.905 & -0.732 & 3.65 & 2.14 & 5.07 & 2.97 & 0.81 & 3.34 & 1.09 & $\mathrm{~B}, \mathrm{MB}$ \\
\hline 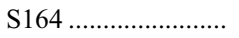 & 303.867 & -0.785 & 1.04 & 0.83 & 1.28 & 1.03 & 0.60 & 1.04 & 0.22 & $\mathrm{~B}, \mathrm{~m}(\mathrm{~S} 163)$ \\
\hline 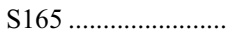 & 303.341 & -0.719 & 0.52 & 0.40 & 0.75 & 0.58 & 0.64 & 0.56 & 0.20 & $\mathrm{C}$ \\
\hline 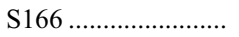 & 302.016 & -0.112 & 0.59 & 0.52 & 0.81 & 0.72 & 0.47 & 0.66 & 0.21 & $\mathrm{C}$ \\
\hline S167 ......................... & 301.625 & -0.347 & 5.82 & 5.34 & 7.30 & 6.69 & 0.40 & 6.28 & 1.42 & $\mathrm{C}$ \\
\hline $\mathrm{S} 168 \ldots \ldots \ldots \ldots \ldots \ldots \ldots$ & 301.548 & 0.300 & 1.21 & 0.88 & 1.78 & 1.29 & 0.69 & 1.27 & 0.48 & $\mathrm{C}$ \\
\hline 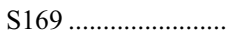 & 301.122 & -0.152 & 2.95 & 2.78 & 4.06 & 3.82 & 0.34 & 3.40 & 1.07 & $\mathrm{~B}$ \\
\hline S170 ........................ & 300.799 & -0.304 & 1.21 & 1.00 & 1.62 & 1.34 & 0.56 & 1.29 & 0.37 & $\mathrm{~B}$ \\
\hline 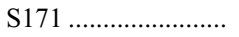 & 300.426 & -0.181 & 0.40 & 0.33 & 0.58 & 0.48 & 0.57 & 0.44 & 0.16 & B \\
\hline 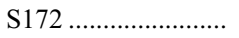 & 299.878 & -0.377 & 1.48 & 1.00 & 1.83 & 1.24 & 0.74 & 1.36 & 0.29 & $\mathrm{C}$ \\
\hline $\mathrm{S} 173 \ldots \ldots$ & 299.776 & -0.286 & 0.67 & 0.38 & 0.89 & 0.50 & 0.83 & 0.59 & 0.16 & $\mathrm{~B}$ \\
\hline 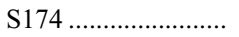 & 299.694 & 0.009 & 2.73 & 2.36 & 3.38 & 2.92 & 0.50 & 2.84 & 0.60 & $\mathrm{C}$ \\
\hline 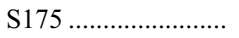 & 299.281 & -0.287 & 1.96 & 1.83 & 2.52 & 2.35 & 0.36 & 2.16 & 0.54 & $\mathrm{C}$ \\
\hline S176 „....................... & 298.941 & -0.687 & 1.23 & 0.93 & 1.94 & 1.46 & 0.66 & 1.38 & 0.62 & $\mathrm{C}$ \\
\hline S177 ......................... & 298.731 & -0.438 & 0.56 & 0.47 & 0.77 & 0.64 & 0.55 & 0.61 & 0.19 & $\mathrm{~B}$ \\
\hline 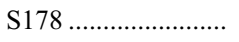 & 298.419 & -0.746 & 1.50 & 1.00 & 1.90 & 1.26 & 0.75 & 1.38 & 0.33 & $\mathrm{~B}, \mathrm{MB}$ \\
\hline S179 „.................... & 298.412 & -0.531 & 0.80 & 0.70 & 1.03 & 0.89 & 0.50 & 0.85 & 0.21 & B \\
\hline S180 ........................ & 298.411 & -0.736 & 0.12 & 0.11 & 0.18 & 0.17 & 0.38 & 0.14 & 0.06 & $\mathrm{C}, \mathrm{CS}, \mathrm{m}(\mathrm{S} 178)$ \\
\hline 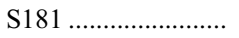 & 298.210 & -0.315 & 1.49 & 1.17 & 1.87 & 1.47 & 0.62 & 1.49 & 0.33 & $\mathrm{C}$ \\
\hline S182 ….................... & 298.186 & -0.220 & 0.93 & 0.74 & 1.13 & 0.89 & 0.61 & 0.92 & 0.17 & B \\
\hline 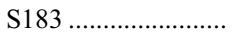 & 297.649 & -0.984 & 0.70 & 0.62 & 0.91 & 0.81 & 0.46 & 0.76 & 0.20 & $\mathrm{C}, \mathrm{BP}$ \\
\hline S184 ........................ & 297.646 & -0.959 & 0.98 & 0.65 & 1.23 & 0.81 & 0.75 & 0.90 & 0.20 & $\mathrm{C}, \mathrm{BP}$ \\
\hline S185 „....................... & 296.563 & -0.972 & 1.41 & 0.89 & 1.83 & 1.15 & 0.78 & 1.29 & 0.33 & $\mathrm{C}$ \\
\hline S186 ........................ & 295.223 & -0.665 & 5.36 & 4.36 & 6.93 & 5.64 & 0.58 & 5.54 & 1.42 & B \\
\hline 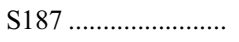 & 295.189 & 0.122 & 0.65 & 0.52 & 0.96 & 0.77 & 0.60 & 0.72 & 0.28 & $\mathrm{C}$ \\
\hline $\mathrm{S} 188 \ldots \ldots \ldots \ldots \ldots \ldots \ldots \ldots$ & 295.030 & -0.565 & 4.48 & 3.29 & 5.76 & 4.23 & 0.68 & 4.39 & 1.10 & B \\
\hline
\end{tabular}

Note.-Table 3 is also available in machine-readable form in the electronic edition of the Astrophysical Journal.

a See footnote to Table 2 . 

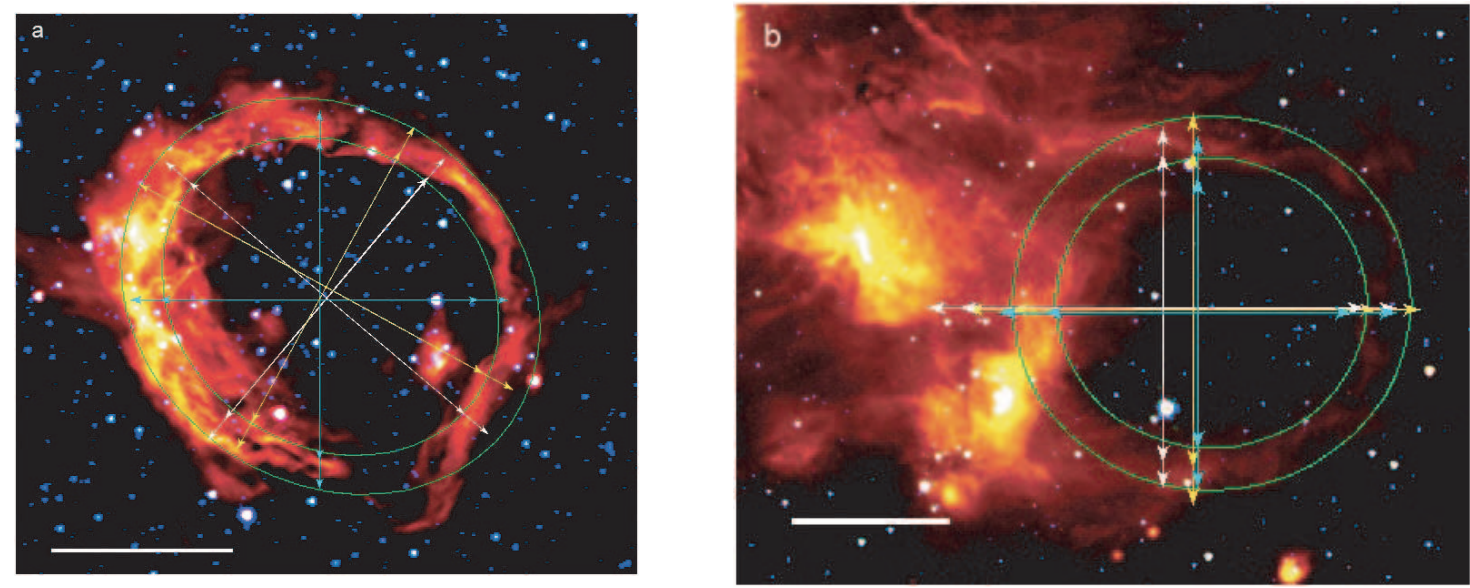

FIG. 1.- (a) Illustration of a bubble (N4) where all four examiners agree on the extent, thickness, and eccentricity. There was disagreement on the orientation of the major axis, but this was because the bubble is almost circular. (b) Illustration of a bubble (S175) where the examiners had substantial disagreements on the size, thickness, and eccentricity. The disagreement was caused mainly by the bright emission to the left of the bubble. In both panels, the annular ellipses fitted by examiner 1 are shown in green. The white, cyan, and yellow arrows show the extent of the major and minor axes of the inner and outer ellipses as measured independently by the other three examiners. The horizontal scale bars represent $2^{\prime}$.

stars, whereas the $8.0 \mu \mathrm{m}$ emission is faint or absent inside the bubbles, is bright along the shell that defines the bubbles, and generally extends well beyond the bubble shell boundary. We have examined numerous images constructed using this combination of wavelengths and find that Figure 3 is representative of the distribution of emission with wavelength in the bubbles so far examined.

To explain the observed difference in distribution of the 8 and $24 \mu \mathrm{m}$ emission, we postulate that the $24 \mu \mathrm{m}$ band is probably dominated by hot thermal dust emission plus perhaps a small contribution from small grains that are transiently heated due to their proximity to one or more hot stars. The $8 \mu \mathrm{m}$ band is probably dominated by the 7.7 and $8.6 \mu \mathrm{m}$ features often attributed to PAHs. The $24 \mu \mathrm{m}$ emission near the hot central star(s) is probably due to silicate grains since small PAHs do not seem to survive in these environments (see below). According to Li \& Draine (2001), no more than $\sim 10 \%$ of interstellar Si can be contained in very small silicate grains, implying an approximately corresponding fraction of their contribution at $24 \mu \mathrm{m}$.

Within some of the bubbles, the $24 \mu \mathrm{m}$ emission seems to peak around a point near the center of the bubble, presumably the central star or cluster responsible for producing the bubble. This morphology is not expected if the $24 \mu \mathrm{m}$-emitting grains are located in an optically thin shell along the inner boundary of the $8 \mu \mathrm{m}$ bubble. This raises two important issues: (1) how the dust is able to survive the intense radiation field produced by the central star or cluster, and (2) why the dust has not been evacuated by the wind(s) of the central star(s). It is difficult to imagine that the bubbles are static. The strong winds of the central O and $\mathrm{B}$ stars, along with overpressure from ionization and high temperature, should cause the dust shells to expand.

The shell thickness of dynamically blown bubbles may have a wide range of values depending on the type of central star, its age, and the density of the ambient ISM. The Weaver et al. (1977, hereafter W77) theory predicts that the ratio of shell thickness to radius increases with both age and ambient interstellar density. At a given age, this ratio increases as the stellar wind luminosity decreases. For example, at an age of $10^{6} \mathrm{yr}$ and an ambient ISM of density $1 \mathrm{~cm}^{-3}$, the W77 theory predicts a thickness-to-radius ratio for an $\mathrm{O} 7 \mathrm{~V}$ star of $\sim 0.07$, but for a B3 V star it is $\sim 0.29$. At an age of $10^{7} \mathrm{yr}$, these ratios increase to $\sim 0.22$ and $\sim 0.33$, respec- tively. The W77 theory suggests that it may not be easy to distinguish between possible static versus expanding bubbles by shell thickness alone. Some images suggest that the $24 \mu \mathrm{m}$ emission is mostly located in a thick shell along the inner boundary of the $8 \mu \mathrm{m}$ shell (Fig. $3 a$ ), but not all. To determine if those bubbles whose $24 \mu \mathrm{m}$ emission peaks at the center are static (not expanding) will require high spatial and velocity resolution line images, preferably of both the ionized and molecular gas associated with the bubbles.

The fact that $8 \mu \mathrm{m}$ emission appears to be essentially absent in the central regions of the bubbles (the front and back sides of the shell that defines the bubble contribute some emission) implies that PAHs are either easily destroyed in the hard radiation field of the central star(s) or blown out of the central regions by stellar winds. Also, the images clearly show that the $8 \mu \mathrm{m}$ emission extends well beyond the obvious dust shell into the photodissociation regions (PDRs) of the bubbles (Hollenbach \& Tielens 1997 and references therein). This strongly supports the notion that PAHs are destroyed in the vicinity of hot stars and instead trace the PDR regions in the neighborhood of hot stars or clusters.

\subsection{Bubble Distribution with Galactic Longitude}

Tables 2 and 3 contain a total of 322 partial or complete rings (bubbles) found in the GLIMPSE survey. This represents an average of about 1.5 bubbles per square degree in the survey area. This is a lower limit due to the selection biases discussed in $\S 2.1$; the true density could be substantially higher. The distribution of the number of bubbles in $2^{\circ} .5$ bins with angular distance $(\Delta l)$ from the Galactic center is shown in Figure 4. The distribution shows no significant dependence on angular distance from the Galactic center in either the north or the south. One might naively expect the surface density of bubbles to decrease with increasing angular distance from the Galactic center because of the decreasing line of sight through the Galaxy. However, there are two selection effects that work against this: (1) more distant bubbles are veiled by foreground diffuse emission, basically limiting the number of bubbles to those at the near kinematic distance or $\lesssim 8 \mathrm{kpc}$ (see Table 4); and (2) an increasingly brighter and spatially variable background makes bubble detection increasingly difficult closer to the Galactic center. In the inner Galaxy, the 

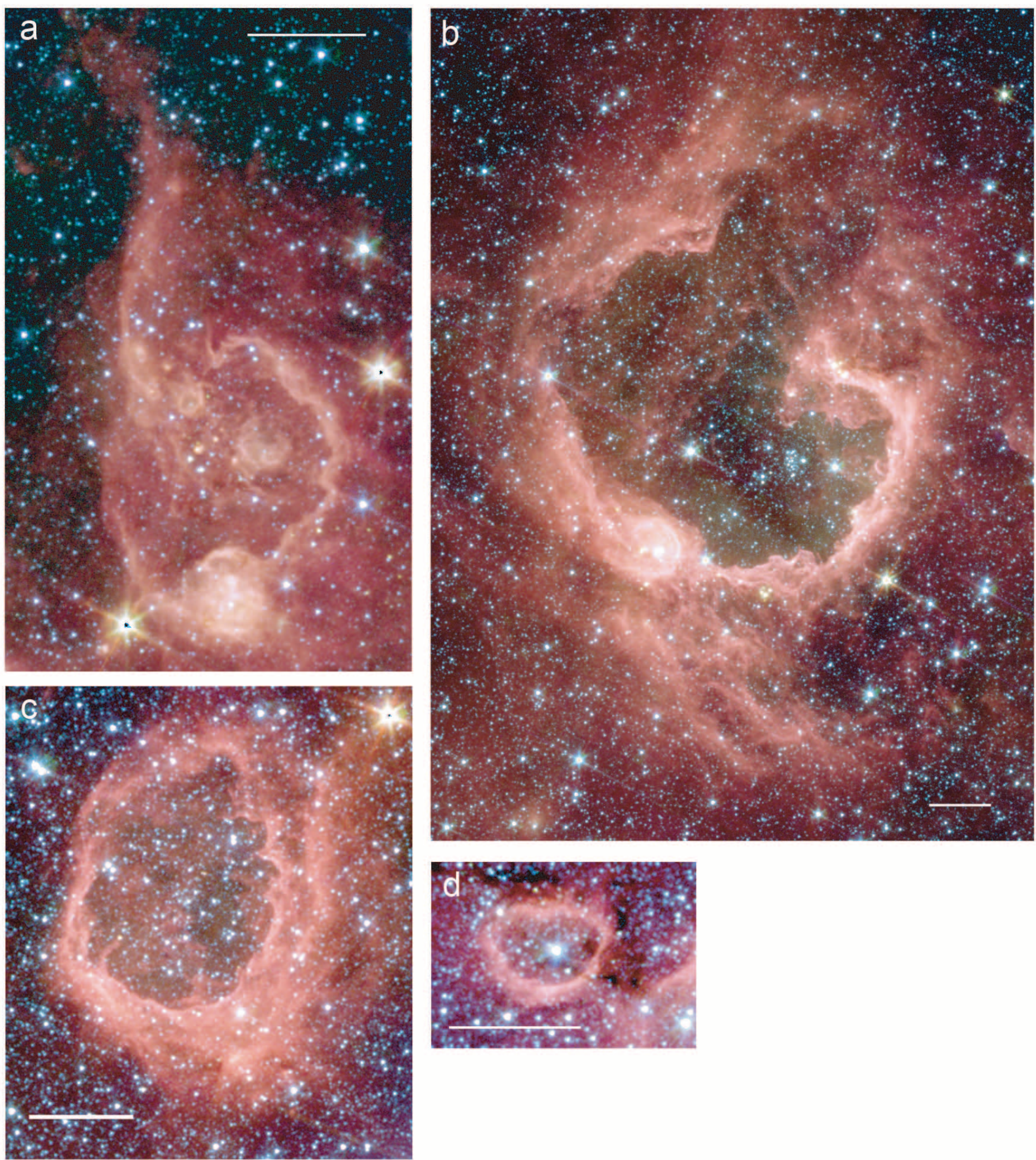

FIG. 2.- Images presented in this montage illustrate all of the bubble morphologies discussed in the text and noted in col. (11) of Tables 2 and 3. The horizontal bar in each panel represents an angular size of $2^{\prime}$. The bubbles shown (by catalog number) are (a) S133, a multiple bubble (MB); (b) S145 (RCW 79), a broken bubble (B) and a multiple bubble (MB) apparently enclosing a star cluster (CC); (c) S44, a complete bubble (C); (d) S21, a complete bubble (C) with a central star $(\mathrm{CS}) ;(e) \mathrm{S} 156$, which has a central star cluster (CC); $(f) \mathrm{S} 97+\mathrm{S} 98$, a bipolar bubble (BP); $(g) \mathrm{S} 109+\mathrm{S} 110+\mathrm{S} 111$, a tripolar bubble (TP); and $(h) \mathrm{S} 100$, a flocculent bubble $(\mathrm{Fl})$. 

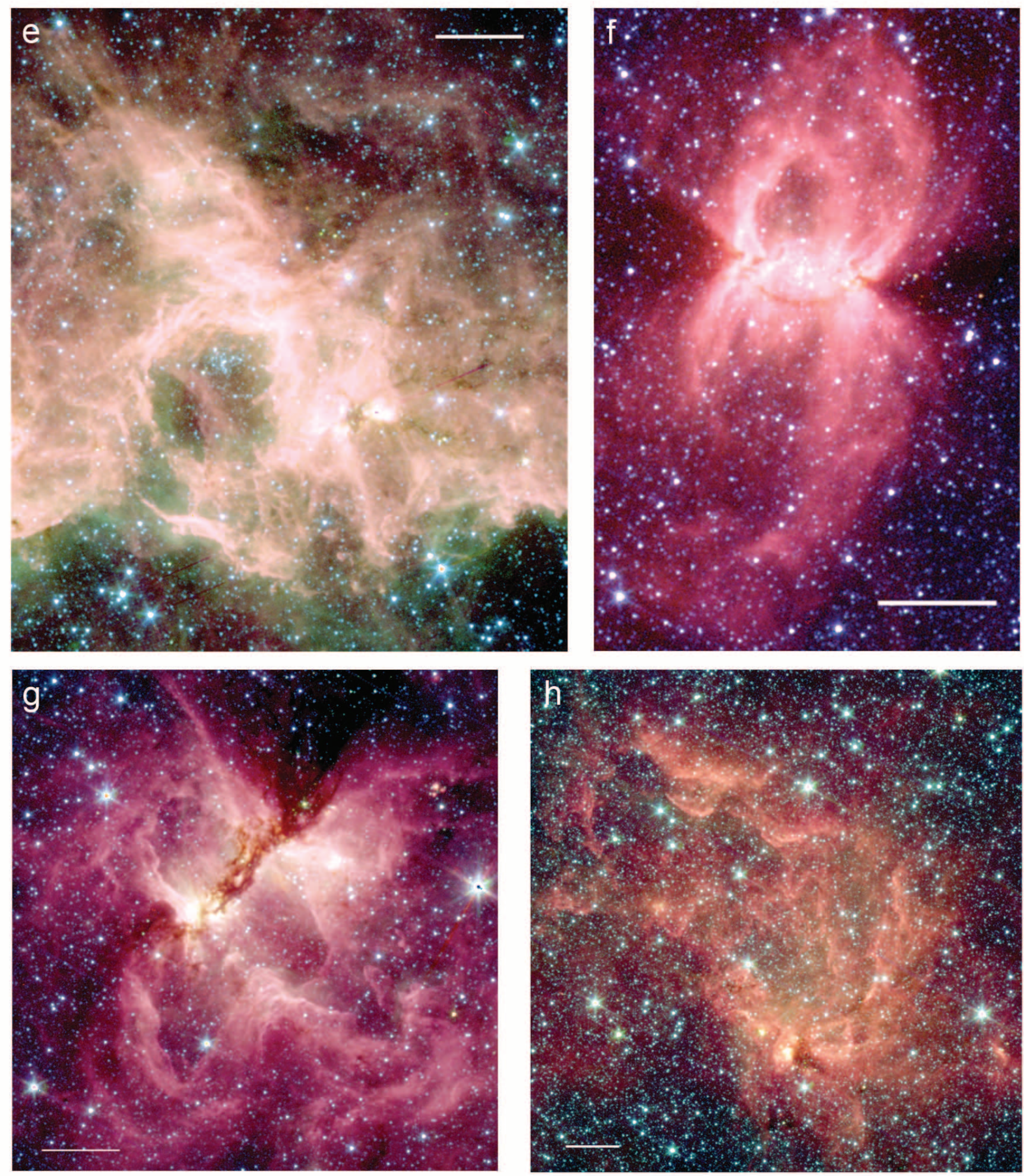

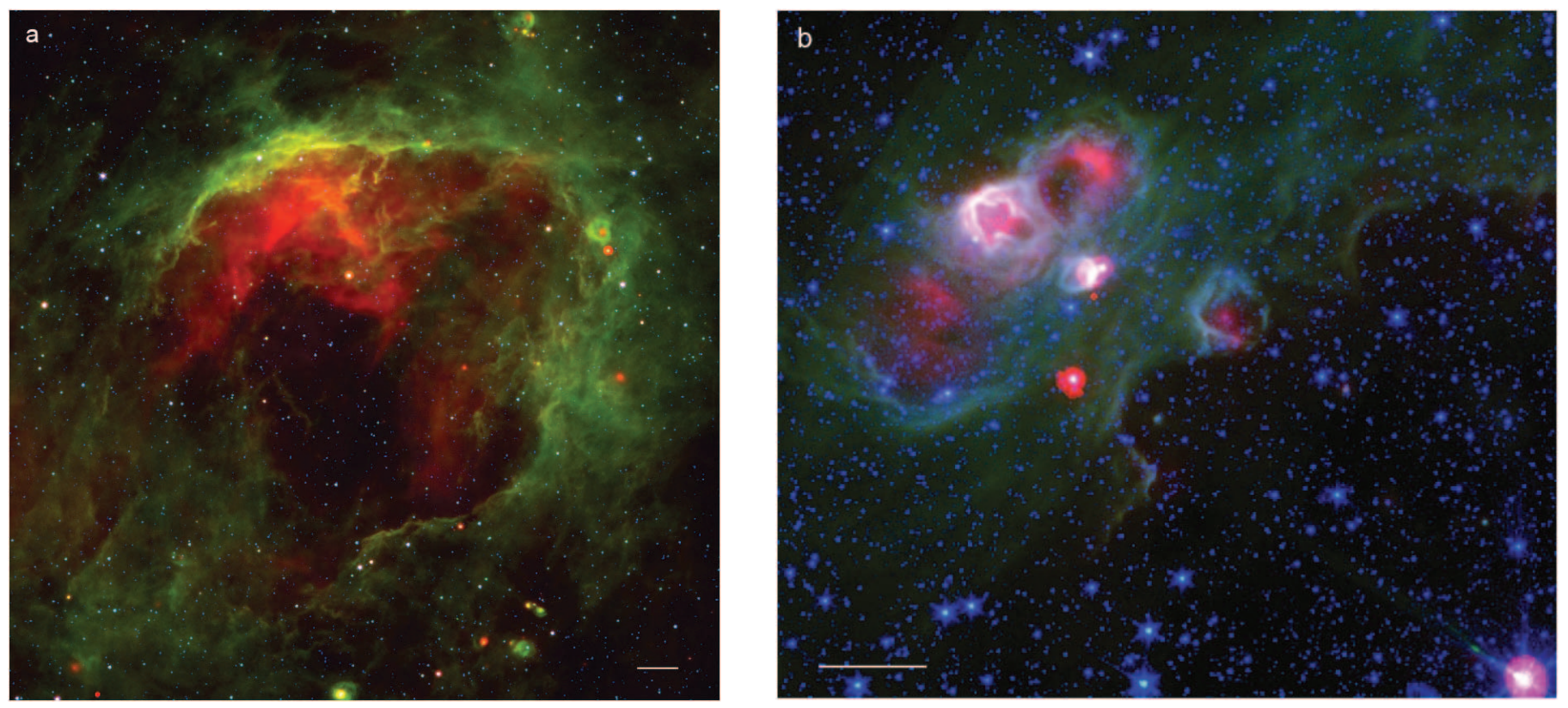

Fig. 3.-These images of (a) large bubble N107 and (b) subsidiary multibubbles N110-N113 on the rim of large MB N109 illustrate the confinement of $24 \mu \mathrm{m}$ emission (red) within the central regions of the bubbles. The $8 \mu \mathrm{m}$ emission (green) delineates the boundaries of the bubbles and generally extends far beyond the bubbles. Blue represents $4.5 \mu \mathrm{m}$ emission. The horizontal scale bars are $2^{\prime}$.

difference between near and far kinematic distances is quite large, making detection of bubbles at the far distance quite unlikely. Thus, the number and surface density of bubbles are surely underestimated, especially for $\Delta l \lesssim 30^{\circ}$ from the Galactic center.

The southern half of the plane contains $59 \%$ of the bubbles. The asymmetry in the number of bubbles in the northern versus the southern halves of the plane appears to be real and probably reflects the fact that massive star formation regions are more numerous in the south. The asymmetry in the number of massive star formation regions was established more than 30 years ago by radio continuum and radio recombination line surveys (see references in Paladini et al. 2004).

Asymmetries between the northern and southern plane in the distribution of molecular clouds (Dame et al. 1987 and others) and $\mathrm{H}$ I (Burton 1985; Kerr et al. 1986) have been known for at least 20 years. Mercer et al. (2005) also found more than twice as many clusters in the southern half of the plane than in the north-

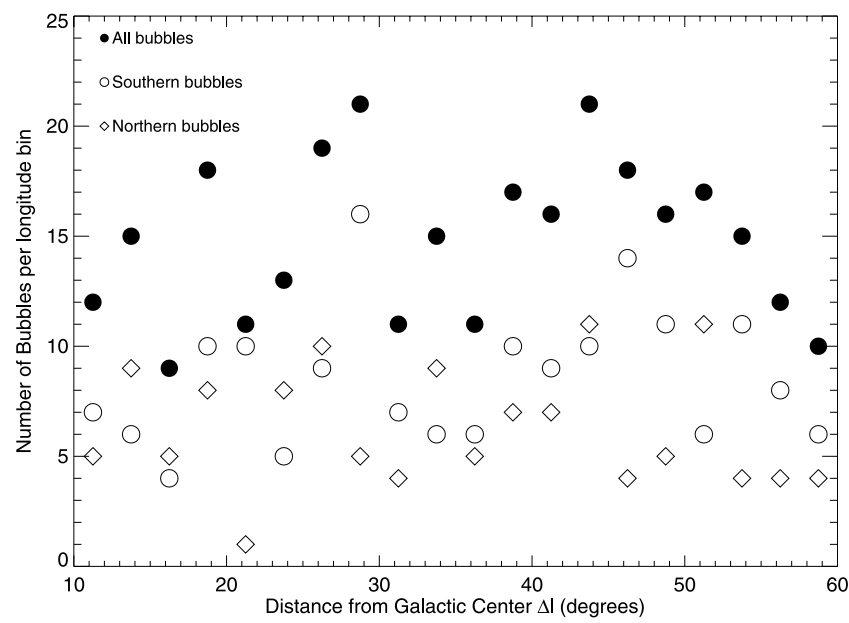

FIG. 4.-Distribution of mid-infrared bubbles as a function of angular distance $\Delta l$ from the Galactic center. There are no obvious trends. ern half. Together, these results lend strong support to our hypothesis that the bubbles in our sample are produced by young $\mathrm{O}$ and $\mathrm{B}$ stars that are known to form in reservoirs of $\mathrm{CO} / \mathrm{H}$ I cloud complexes. If the bubbles are primarily produced by young $\mathrm{OB}$ stars, then the bubble distribution in the inner Galaxy predicts at least 1.5 massive star formation regions per square degree within a distance of $\lesssim 8 \mathrm{kpc}$.

\subsection{Bubble Distribution with Galactic Latitude}

The distribution of bubbles with latitude (all observed bubbles within a given latitude bin have been summed over all observed longitudes) is plotted in Figure 5. The distributions of those in the southern and northern segments of the plane are also shown in Figure 5. All three curves show that the bubbles are concentrated toward the midplane, but the peak is located at $b=-0^{\circ} .08 \pm 0^{\circ} .03$ for all bubbles, $b=-0^{\circ} .14 \pm 0.04$ for the southern plane, and $b=-0.02 \pm 0.04$ for the northern plane, where the errors are $1 \sigma$ calculated from the quality of Gaussian fits to the data.

We find angular scale heights of $0.63 \pm 0.03,0.67 \pm 0.04$, and $0.61 \pm 0.05$ for all bubbles, southern plane bubbles, and northern plane bubbles, respectively. This is equivalent to a scale height of $44 \mathrm{pc}$ at a distance of $4 \mathrm{kpc}$. For comparison, Becker et al. (1990) found a scale height of $25 \mathrm{pc}$ for small (angular diameters between $3^{\prime \prime}$ and $20^{\prime \prime}$ ) radio sources at longitudes within $50^{\circ}$ of the Galactic center. They argued that the sources are compact or ultracompact $\mathrm{H}$ II regions ionized by extreme Population I stars. Wood \& Churchwell (1989) found a scale height of $0.60 \pm 0.05$ for a sample of 1717 far-infrared color-selected $\mathrm{H}$ II region candidates from the Infrared Astronomical Satellite (IRAS) catalog distributed over the entire range of Galactic longitudes and latitudes. Gilmore \& Zeilik (2000) give a scale height of $60 \mathrm{pc}$ for extreme Population I stars in the inner Galaxy. Because the bubble angular scale height is similar to that of $\mathrm{H}$ II regions and substantially less than that of planetary nebulae ( $\sim 500 \mathrm{pc}$; Becker et al. 1990) and SNRs ( 130 pc; Becker et al. 1990), it strongly supports the identification of the bubbles in GLIMPSE with $\mathrm{H}$ II regions and a young $\mathrm{OB}$ star population. 
TABLE 4

Sizes and Thicknesses of Bubbles with Known Kinematic Distances

\begin{tabular}{|c|c|c|c|c|c|c|c|c|}
\hline \multirow[b]{2}{*}{$\begin{array}{c}\text { CAtalog Number } \\
\text { (1) }\end{array}$} & \multicolumn{4}{|c|}{ Near Kinematic Distance ${ }^{a}$} & \multicolumn{4}{|c|}{ Far Kinematic Distance } \\
\hline & $\begin{array}{c}D \\
(\mathrm{kpc}) \\
(2)\end{array}$ & $\begin{array}{c}\delta D \\
(\mathrm{kpc}) \\
(3)\end{array}$ & $\begin{array}{c}\langle R\rangle \\
(\mathrm{pc}) \\
(4)\end{array}$ & $\begin{array}{c}\langle T\rangle \\
(\mathrm{pc}) \\
(5)\end{array}$ & $\begin{array}{c}D \\
(\mathrm{kpc}) \\
(6)\end{array}$ & $\begin{array}{c}\delta D \\
(\mathrm{kpc}) \\
(7)\end{array}$ & $\begin{array}{c}\langle R\rangle \\
(\mathrm{pc}) \\
(8)\end{array}$ & $\begin{array}{c}\langle T\rangle \\
\text { (pc) } \\
\text { (9) }\end{array}$ \\
\hline $2 \ldots$ & 8.4 & 3.5 & 16.9 & 3.0 & & & & \\
\hline $\mathrm{N} 4 \ldots$ & 3.2 & 0.9 & 1.9 & 0.4 & 13.4 & 0.9 & 7.8 & 1.7 \\
\hline N5.. & 4.2 & 0.6 & 4.0 & 0.8 & 12.4 & 0.6 & 11.7 & 2.4 \\
\hline $\mathrm{N} 10 \ldots$ & 4.9 & 0.5 & 1.7 & 0.4 & 11.7 & 0.5 & 4.1 & 0.9 \\
\hline $\mathrm{N} 14 \ldots \ldots \ldots \ldots \ldots \ldots$ & 3.7 & 0.6 & 1.3 & 0.4 & 12.8 & 0.6 & 4.5 & 1.4 \\
\hline 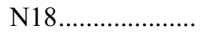 & 3.9 & 0.5 & 8.3 & 1.9 & 12.3 & 0.5 & 26.0 & 6.1 \\
\hline $\mathrm{N} 21 \ldots$ & 3.7 & 0.6 & 2.3 & 0.5 & 12.4 & 0.6 & 7.8 & 1.8 \\
\hline $\mathrm{N} 22$. & 4.1 & 0.5 & 2.0 & 0.5 & 12.1 & 0.5 & 5.9 & 1.5 \\
\hline $\mathrm{N} 24^{\mathrm{b}}$ & 4.8 & 0.4 & 11.0 & 3.9 & 11.3 & 0.4 & 26.2 & 9.2 \\
\hline N30. & 2.5 & 0.7 & 0.8 & 0.2 & 13.1 & 0.7 & 4.1 & 1.3 \\
\hline $\mathrm{N} 32$. & 4.6 & 0.5 & 0.6 & 0.2 & 11.0 & 0. & 1.3 & 0.4 \\
\hline N35. & 6.9 & 0.7 & 6.5 & 1.9 & 8.6 & 0 . & 8.1 & 2.3 \\
\hline N36. & 6.5 & 0.7 & 5.3 & 1.6 & 8.9 & 0. & 7.3 & 2.3 \\
\hline N37. & 3.1 & 0.6 & 1.6 & 0.4 & 12.3 & 0.6 & 6.3 & 1.7 \\
\hline N39. & 4.1 & 0.5 & 2.4 & 0.8 & 11.3 & 0. & 6.5 & 2.1 \\
\hline $\mathrm{N} 45$. & 5.0 & 0.5 & 2.2 & 0.8 & 10.1 & 0 & 4.3 & 1.6 \\
\hline $\mathrm{N} 47 .$. & 6.2 & 0.8 & 4.0 & 0.9 & 8.8 & 0.8 & 5.7 & 1.3 \\
\hline $\mathrm{N} 49 \ldots \ldots$. & 5.7 & 0.6 & 2. & 0.5 & 9.2 & 0.6 & 3.5 & 0.9 \\
\hline $\mathrm{N} 52 \ldots$ & 5.9 & 1. & 3. & 1 & 8.7 & 1.0 & 5.2 & 1.6 \\
\hline $\mathrm{N} 59 \ldots$ & 6.6 & 0.8 & 12.9 & 2.6 & 7.6 & 0.8 & 14.8 & 3.0 \\
\hline N64.. & 3.6 & 0 & 5 & 1.0 & 10.4 & 0. & 14.5 & 3.0 \\
\hline $\mathrm{N} 68^{\mathrm{c}}$. & 3.6 & 0.6 & 4.9 & 0.8 & 10.2 & 0.6 & 13.9 & 2.2 \\
\hline 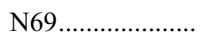 & 5.3 & 1. & 14.4 & 2 & 8.4 & 1.2 & 22.7 & 4.3 \\
\hline $\mathrm{N} 70 \ldots \ldots$. & 4.0 & 0. & 0 & 0 & 9.4 & 0 & 1.5 & 0.5 \\
\hline $\mathrm{N} 79 \ldots$ & 1.3 & 0.7 & 0.5 & 0.1 & 11.4 & 0.7 & 4.2 & 1.0 \\
\hline $\mathrm{N} 82 \ldots$ & 5.3 & 1. & 2.6 & 0.7 & 7.3 & 1.1 & 3.6 & 0.9 \\
\hline $\mathrm{N} 91 \ldots$ & 4.9 & 1.1 & 7.3 & 2.4 & 7.3 & 1.1 & 10.7 & 3.6 \\
\hline N94.... & 3.4 & 0.9 & 3.7 & 1.2 & 8.6 & 0.9 & 9.4 & 2.9 \\
\hline $\mathrm{N} 107^{\mathrm{c}}$ & 4.7 & 0.8 & 15.5 & 3.1 & 6.0 & 0.8 & 19.9 & 3.9 \\
\hline N113 ....................... & 0.2 & 2.1 & 0.0 & 0.0 & 10.2 & 2.1 & 1.7 & 0.7 \\
\hline N117.. & 5.0 & 1.0 & 2.4 & 0.7 & $\ldots$ & $\cdots$ & $\ldots$ & . \\
\hline N123... & 0.3 & 1.7 & 0.1 & 0.0 & 8.9 & 1.7 & 3.6 & 0.8 \\
\hline $\mathrm{N} 133 \ldots$ & 2.5 & 1.4 & 1.2 & 0.3 & 5.2 & 1.4 & 2.5 & 0.6 \\
\hline $\mathrm{N} 134 \ldots$ & 3.7 & 1.1 & 0.6 & 0.3 & $\ldots$ & $\ldots$ & $\ldots$ & $\ldots$ \\
\hline $\mathrm{S} 1^{\mathrm{c}} \ldots$ & 3.6 & 0.9 & 4.7 & 1.1 & 13.2 & 0.9 & 17.3 & 4.2 \\
\hline S7 ... & $\leq 3.3$ & 3.3 & $\leq 4.3$ & $\leq 0.8$ & 16.6 & 3.3 & 21.6 & 3.8 \\
\hline S8 ... & 6.3 & 0.3 & 3.1 & 0.6 & 10.2 & 0.3 & 5.0 & 1.0 \\
\hline S15. & 3.1 & 0. & .9 & 0.9 & 3.2 & 0.7 & 8.1 & 3.7 \\
\hline S20. & 1.0 & 3.1 & 1.4 & 0.1 & 15.2 & 3.1 & 5.5 & 1.9 \\
\hline S29 ... & 3.3 & 0 & 2.5 & 0.5 & 12.6 & 0 & 9.6 & 1.8 \\
\hline 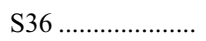 & 4.0 & 0 & 3. & 0.8 & 11.7 & 0 & 9.3 & 2.4 \\
\hline S44 ........ & 4.6 & 0. & 3 & 0.7 & 10.8 & 0.5 & 7.4 & 1.6 \\
\hline S57. & 5.1 & 0 . & 5.7 & 1.1 & 99 & 0.5 & 11.0 & 2.2 \\
\hline S62. & 4.4 & 0. & 2. & 0.6 & 10.5 & 0. & 6.2 & 1.4 \\
\hline S64 ........................... & 3.8 & 0 . & 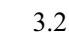 & 0.8 & 11.0 & 0.5 & 9.2 & 2.4 \\
\hline S67 ....... & 4.9 & 0.5 & 1.4 & 0.5 & 10.0 & 0.5 & 2.8 & 0.9 \\
\hline 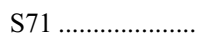 & 7.2 & 0.6 & 2.2 & 0.6 & $\ldots$ & $\ldots$ & $\ldots$ & $\ldots$ \\
\hline $\mathrm{S} 76^{\mathrm{c}} .$. & 3.4 & 0.6 & 4.8 & 1.1 & 10.9 & 0.6 & 15.6 & 3.7 \\
\hline S96.. & 2.6 & 0.7 & 1.1 & 0.3 & 10.4 & 0.7 & 4.3 & 1.2 \\
\hline S97 ....... & 2.7 & 0.7 & 1.2 & 0.4 & 10.3 & 0.7 & 4.5 & 1.6 \\
\hline S104 ......................... & 2.7 & 0.7 & 1.4 & 0.5 & 9.9 & 0.7 & 5.0 & 2.0 \\
\hline S110 ....................... & 2.7 & 0.7 & 1.1 & 0.3 & 9.7 & 0.7 & 4.0 & 1.0 \\
\hline S116 ............................. & 5.9 & 0.9 & 7.2 & 0.9 & $\ldots$ & $\ldots$ & $\ldots$ & $\ldots$ \\
\hline S123 ……............... & 3.9 & 1.4 & 2.6 & 0.6 & 7.7 & 1.4 & 5.2 & 1.2 \\
\hline $\mathrm{S} 133 \ldots$ & 5.6 & 0.3 & 3.3 & 0.9 & . & $\ldots$ & $\ldots$ & $\ldots$ \\
\hline $\mathrm{S} 134 \ldots . .$. & 5.6 & 0.3 & 1.0 & 0.3 & $\ldots$ & & . & \\
\hline $\mathrm{S} 136^{\mathrm{c}} \ldots \ldots$ & 5.5 & 0.9 & 1.0 & 0.3 & .. & & $\cdots$ & $\cdots$ \\
\hline $137 \ldots$ & 5.1 & 1.1 & 4.6 & 1.4 & 6.0 & 1.1 & 5.5 & 1.6 \\
\hline $\mathrm{S} 141 \ldots$ & 3.9 & 1.3 & 1.7 & 0.4 & 6.9 & 1.3 & 2.9 & 0.7 \\
\hline S143. & 5.3 & 1.1 & 7.2 & 1.3 & & & & \\
\hline $\mathrm{S} 145^{\mathrm{c}} \ldots \ldots \ldots \ldots \ldots$ & 5.3 & 0.9 & 8.9 & 1.6 & & & & \\
\hline
\end{tabular}

TABLE 4-Continued

\begin{tabular}{|c|c|c|c|c|c|c|c|c|}
\hline \multirow[b]{2}{*}{$\begin{array}{c}\text { Catalog Number } \\
\text { (1) }\end{array}$} & \multicolumn{4}{|c|}{ Near Kinematic Distance ${ }^{a}$} & \multicolumn{4}{|c|}{ Far Kinematic Distance } \\
\hline & $\begin{array}{c}D \\
(\mathrm{kpc}) \\
(2)\end{array}$ & $\begin{array}{c}\delta D \\
(\mathrm{kpc}) \\
(3)\end{array}$ & $\begin{array}{c}\langle R\rangle \\
(\mathrm{pc}) \\
(4)\end{array}$ & $\begin{array}{c}\langle T\rangle \\
(\mathrm{pc}) \\
(5)\end{array}$ & $\begin{array}{c}D \\
(\mathrm{kpc}) \\
(6)\end{array}$ & $\begin{array}{c}\delta D \\
(\mathrm{kpc}) \\
(7)\end{array}$ & $\begin{array}{c}\langle R\rangle \\
(\mathrm{pc}) \\
(8)\end{array}$ & $\begin{array}{c}\langle T\rangle \\
\text { (pc) } \\
\text { (9) }\end{array}$ \\
\hline S149. & 4.9 & 0.7 & 0.6 & 0.3 & $\cdots$ & $\ldots$ & . & \\
\hline $\mathrm{S} 156 \ldots \ldots$. & 4.9 & 1.1 & 2.7 & 0.8 & $\ldots$ & $\ldots$ & $\ldots$ & . \\
\hline $\mathrm{S} 181 \ldots \ldots \ldots \ldots \ldots$ & 4.0 & 0.0 & 1.7 & 0.4 & $\ldots$ & $\ldots$ & $\ldots$ & .. \\
\hline $\mathrm{S} 183 \ldots \ldots \ldots \ldots \ldots$ & 3.9 & 0.0 & 0.9 & 0.2 & $\ldots$ & $\ldots$ & $\ldots$ & 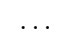 \\
\hline $\mathrm{S} 186 \ldots \ldots \ldots \ldots \ldots \ldots$ & 3.6 & 0.0 & 5.8 & 1.5 & $\ldots$ & $\ldots$ & $\ldots$ & \\
\hline
\end{tabular}

${ }^{\text {a }}$ When a bubble lies at the tangent point, no far kinematic distance is given, and the near kinematic distance is the tangent point distance.

${ }^{b}$ Distance determined from three $\mathrm{H}$ II regions found within the bubble.

${ }^{c}$ Distance determined from two $\mathrm{H}$ II regions found within the bubble.

The offset of the bubble distribution below the midplane may be a transient configuration of the early star population in the inner Galaxy. The location of the midplane, as determined by the distribution of all stars in the GLIMPSE catalog, does not shift from the canonical definition by more than 0.05 (Benjamin et al. 2005), but the latitude distribution of OB stars could be different from that of all stars in the GLIMPSE catalog, 90\% of which are K giants. In fact, Figure $4 a$ of Becker et al. (1990) shows that the positions of compact radio sources with angular diameters $>3^{\prime \prime}$ in the longitude range $-20^{\circ}$ to $+40^{\circ}$ are skewed to negative latitudes. Also, the $\mathrm{H}$ II region candidates in Figure $12 a$ of Helfand et al. (1992) are skewed to negative latitudes. Wood \& Churchwell (1989) found that their sample was centered at $b=0^{\circ}$, but this sample includes the entire plane. We conclude that the OB stars in the inner Galaxy are, on average, offset by $\sim 0.1$ below the midplane. The mean $\mathrm{CO}$ distribution is also known to be offset below the midplane (Cohen \& Thaddeus 1977; Clemens et al. 1988). Mercer et al. (2005) also found $\sim 40 \%$ fewer clusters above the midplane than below it.

None of the bubble parameters (size, thickness, eccentricity, or orientation of the major axis) show any significant dependence on latitude. Since the scale height of CO in the inner Galaxy is only 60-75 pc (Cohen \& Thaddeus 1977; Dame \& Thaddeus 1985), one might expect a possible correlation of both bubble size

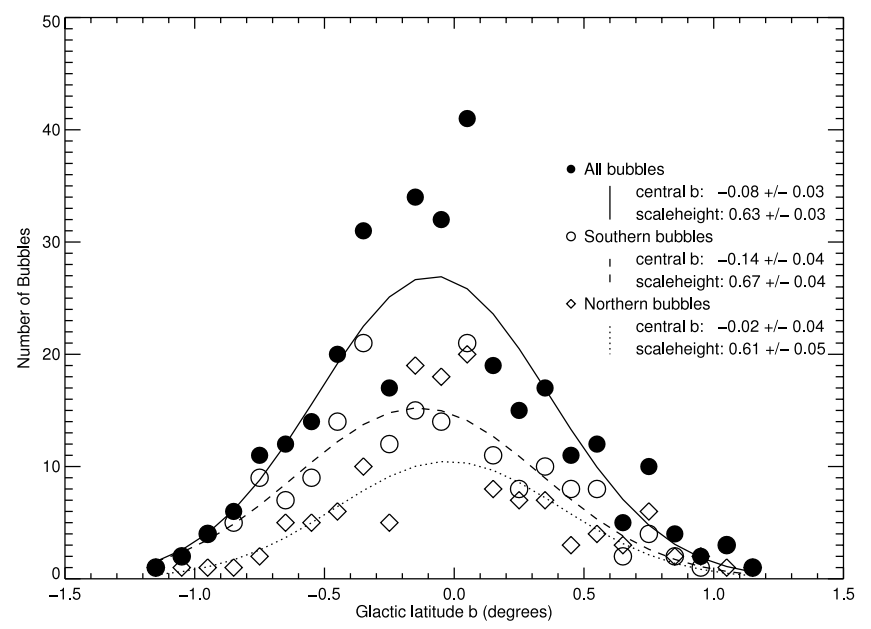

FIG. 5.-Distribution with latitude for the full sample of bubbles, as well as the northern and southern samples separately. The errors are $1 \sigma$ determined from the quality of Gaussian fits to the distributions. 


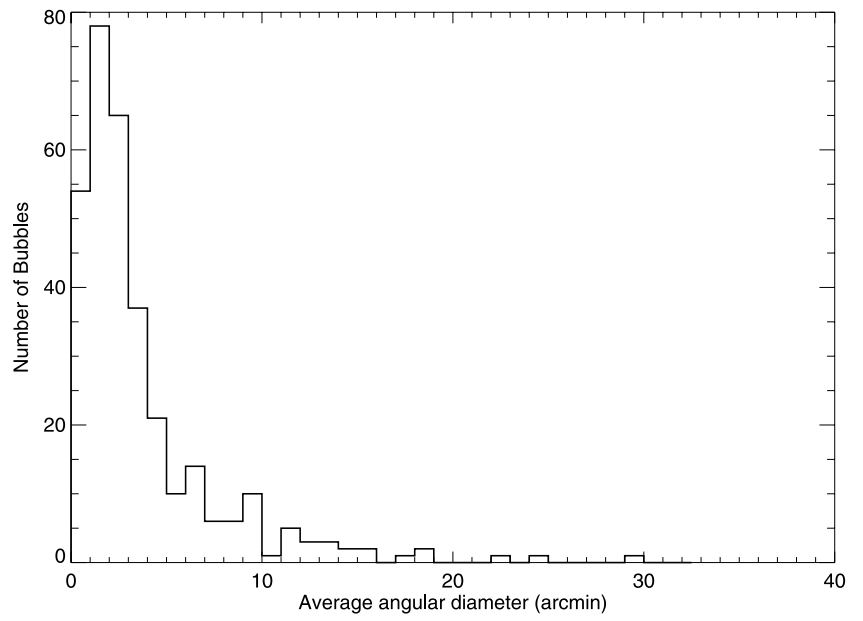

FIG. 6.- Observed distribution of average bubble angular diameters $(2\langle R\rangle)$.

and orientation of the break in incomplete bubbles with distance from the Galactic plane. The fact that we find no significant increase in average bubble size with increasing latitude suggests that the average gradient of ambient interstellar pressure within $1^{\circ}$ of the Galactic plane is less important than the variation of local pressure in the natal cloud into which the bubbles are expanding. We also examined the orientation of breaks (blowouts) in incomplete bubbles and found no significant tendency for the breaks to be oriented away from the Galactic plane. We conclude that bubble size, orientation of breaks in bubbles, and orientation of the major axes of bubbles are functions of stellar wind luminosities and orientations and the local distribution of interstellar matter rather than the average large-scale gradient of the ISM away from the Galactic plane.

\subsection{Bubble Size Distribution}

The distribution of average bubble angular diameters in our sample is shown in Figure 6. The distribution peaks between $1^{\prime}$ and $3^{\prime}$ and falls off sharply at greater and smaller angular diameters. Within the statistical uncertainties, the distribution is the same in the northern and southern segments of the Galactic plane. This is true of all of the distributions discussed below unless otherwise noted.

There are observational biases against bubbles smaller than about $1^{\prime}$ and larger than about $25^{\prime}$. Bubbles smaller than about $30^{\prime \prime}$ are prone to be missed because they have to be substantially brighter than the background to be identified as bubbles. Since the background levels generally increase with decreasing angular distance from the Galactic center, the contrast between bubbles and the background systematically decreases, making detection increasingly difficult in the inner Galaxy. Also, one must zoom in and change the color stretch to identify a small bright patch as a bubble; this inevitable extra image manipulation means that many small bubbles have probably been missed. This is also probably why no planetary nebulae are included in the sample: the planetary nebulae identified in the GLIMPSE survey have small angular sizes and are generally quite faint. Bubbles larger than about $25^{\prime}$ generally have low surface brightness and can also easily be camouflaged by the Galactic background. We are not aware of other biases that might distort the observed size distribution and believe that in the range from $\sim 1^{\prime}$ to $\sim 25^{\prime}$ the observed distribution is likely to be representative of the actual angular size distribution. In this size range, about $98 \%$ of the bubbles in our sample have angular diameters $<10^{\prime}$ and $88 \%$ have angular diameters $\leq 4^{\prime}$.
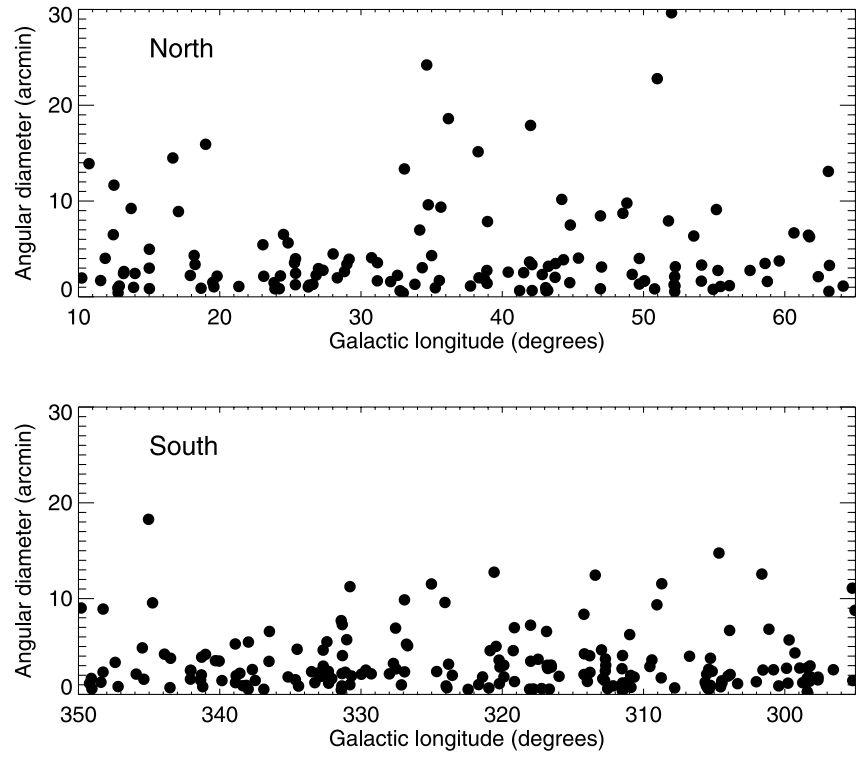

FIG. 7.-Average bubble angular diameters $(2\langle R\rangle)$ as a function of Galactic longitude for both the northern and southern halves of the Galactic plane. Although bubbles are more numerous in the southern plane, nearly all bubbles with angular diameters greater than $15^{\prime}$ are in the northern plane.

Average bubble angular diameters $(2\langle R\rangle)$ as a function of Galactic longitude are plotted in Figure 7 for both the northern and southern halves of the Galactic plane. Figure 7 shows that the range of bubble angular diameters is larger in the northern than in the southern plane. In fact, even though there are fewer bubbles in the northern than in the southern plane, bubbles occupy a larger angular area in the northern than in the southern plane.

Figure 8 shows the average angular diameters of all bubbles as a function of angular distance from the Galactic center (the northern and southern bubbles at the same absolute angular distance from the center are counted together). This figure shows that most bubbles have angular diameters $\leq 5^{\prime}$ at all longitudes. The median and average angular diameters are roughly constant with angular distance from the Galactic center. The upper envelope of bubble angular diameters appears to systematically increase with distance from the Galactic center; however, this trend depends on a small number of objects in the northern plane only, so it is probably not statistically significant. The few largest bubbles are without exception faint and primarily found at large angular distances from the Galactic center where the background becomes faint enough for these bubbles to be detected above the background. Figures 7 and 8 support the hypothesis that the bubbles lie within $\sim 8 \mathrm{kpc}$ and are therefore insensitive to longitude distribution effects.

The physical size of bubbles depends on several parameters: luminosity of the central illuminating star or cluster, the strength of the stellar wind(s) of the central star(s), the density of the ambient ISM, the relative motions of the ambient ISM and the illuminating star(s), the orientation and strength of the magnetic field in the ambient ISM, and the age of the illuminating star(s). Properties of spherical stellar wind-blown bubbles have been investigated by Hollenbach et al. (1976), W77, and others.

\subsection{Projected Bubble Thicknesses}

The distribution of average projected bubble thickness, as defined by equation (2), is plotted in Figure 9. The thickness of $87 \%$ of the sample is less than $1^{\prime}$, and $97.5 \%$ have thicknesses less than $2^{\prime}$. The average distribution of thickness relative to average bubble radius $\left(\langle T\rangle /\left\langle R_{\text {out }}\right\rangle\right)$ is shown in Figure 10 . We see that the ratio ranges from $\sim 0.1$ to $\sim 0.65$, but with nearly $89 \%$ of 


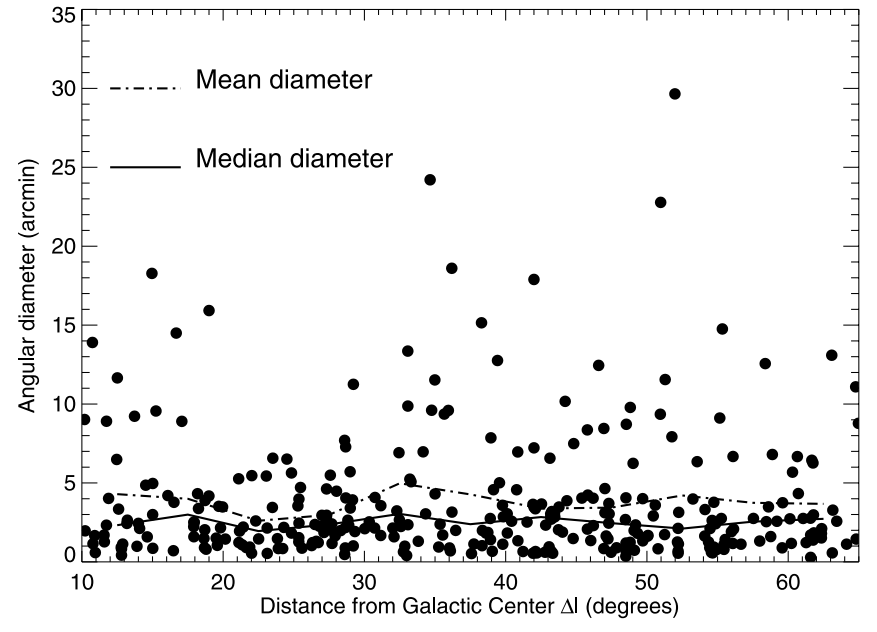

FIG. 8.-Average angular diameters of all bubbles with angular distance $\Delta l$ from the Galactic center. Both the average and median angular diameters are essentially constant with longitude, although the upper envelope of the angular diameters steadily increases with distance from the center.

the sample having thicknesses between 0.2 and 0.4 of their outer radii. Many of the bubble shells exhibit a filamentary structure suggesting a low dust filling factor. The physical thickness of a bubble is strongly dependent on the density of the ambient ISM into which the bubble is expanding, the stellar wind luminosity, and the age of the star or cluster (W77). The observed angular thickness will depend on the distribution of optical depth through the shell and the abundance of PAHs.

We plot shell thickness versus mean radius in angular units for all sources in Tables 2 and 3 in Figure $11 a$, and in Figure $11 b$ the sources in Table 4 are plotted using absolute units assuming near or tangent point kinematic distances. Both plots show that bubble thickness increases linearly with bubble size. This is approximately in agreement with the W77 bubble evolution models shown in Figure 12. The predicted thickness versus radius for a B3 $\mathrm{V}$ star for ambient ISM densities ranging from 0.1 to $10^{4} \mathrm{~cm}^{-3}$ and the same quantities for an $\mathrm{O} 7 \mathrm{~V}$ star are plotted in Figures $12 a$ and $12 b$, respectively. The luminosities are from Vacca et al. (1996) and Cox (2000) for the O7 V and B3 V star, respectively. The mass-loss rates were estimated using equation (11) of Abbot et al. (1981). The W77 models predict that radius increases faster than thickness in the earliest phases of evolution (small radii). This is not obvious in the observed behavior shown in Figure 11. Our

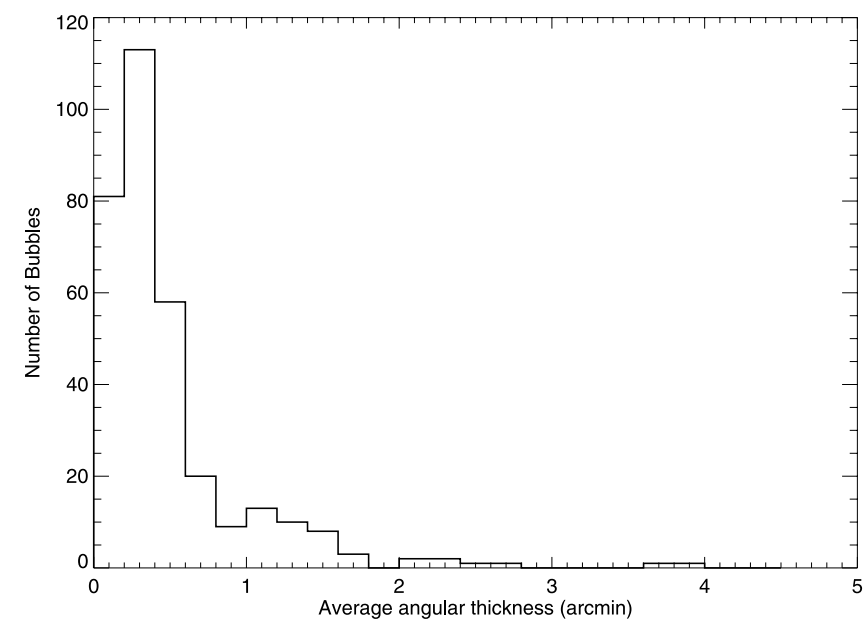

FIG. 9.-Distribution of average bubble thickness (angular measure $\langle T\rangle$ ).

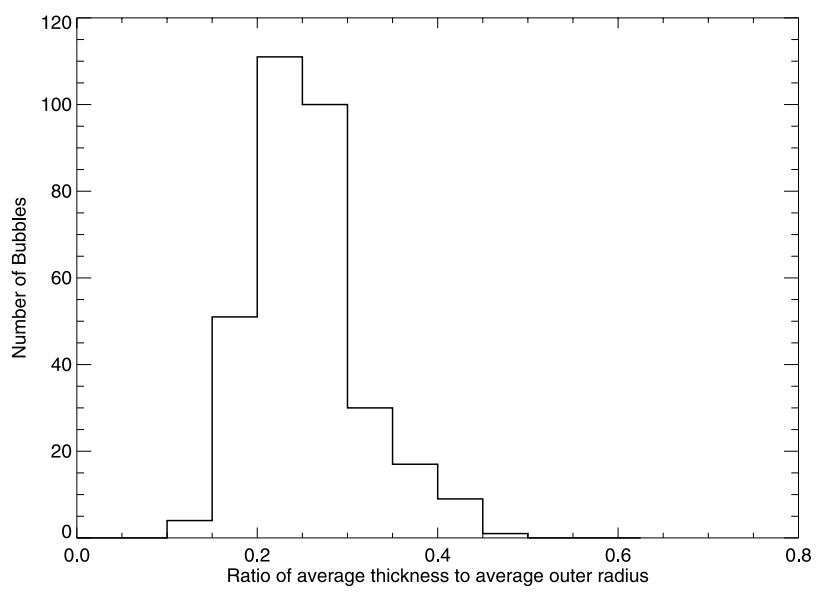

FIG. 10.-Distribution of bubble thickness relative to average outer radius $\left(\langle T\rangle /\left\langle R_{\text {out }}\right\rangle\right)$. Nearly $89 \%$ of the members of the sample have ratios between 0.2 and 0.4 .

sample may include too few very young bubbles in our sample to exhibit this effect. It is also possible that the models require modification. The models assume a uniform ambient ISM density and homogeneous shells, neither of which is true. The GLIMPSE images show quite clearly that the dust shells are filamentary and the ISM is far from uniform. From this perspective, it is perhaps surprising that observations and theory appear to agree reasonably well.

In Figure $11 a$ there are 12 bubbles with mean radii greater than 6.5 , and of these only 2 are in the southern plane, another illustration that most of the largest bubbles are in the northern plane.

\subsection{Bubble Eccentricities and Breaks}

The distribution of bubble eccentricities is shown in Figure 13. This histogram shows a slow rise in eccentricity from near circular to a peak at $\sim 0.65$ with a sharp drop-off at eccentricities greater than 0.85 and less than 0.55 . Eccentricity was calculated as

$$
e=\frac{\left(R^{2}-r^{2}\right)^{1 / 2}}{R}
$$

where $R$ is the semimajor axis and $r$ is the semiminor axis of either the inner or outer ellipse (both the inner and outer ellipses are constrained to have the same eccentricity). The bubbles in the GLIMPSE sample are quite eccentric.

Incomplete or "broken" bubbles, designated " $\mathrm{B}$ " in column (11) of Tables 2 and 3, represent $38 \%$ of the total sample. These are bubbles that probably have met little or no resistance by the ambient ISM in a given direction, although it is also possible that PAH destruction extends further or that UV radiation is attenuated in the direction of a "break." There is no apparent difference between complete and broken bubbles in the distributions discussed above. Broken bubbles, along with the high eccentricities of the bubbles, probably imply that the ISM in the Galactic plane is far from uniform and that the stellar winds and radiation driving the bubbles are anisotropic.

\subsection{Associations of Bubbles with Known Objects}

There are several classes of objects that could produce the bubbles in the GLIMPSE sample. Among these are supernovae, OB stars, Wolf-Rayet (W-R) stars, planetary nebulae, and open 

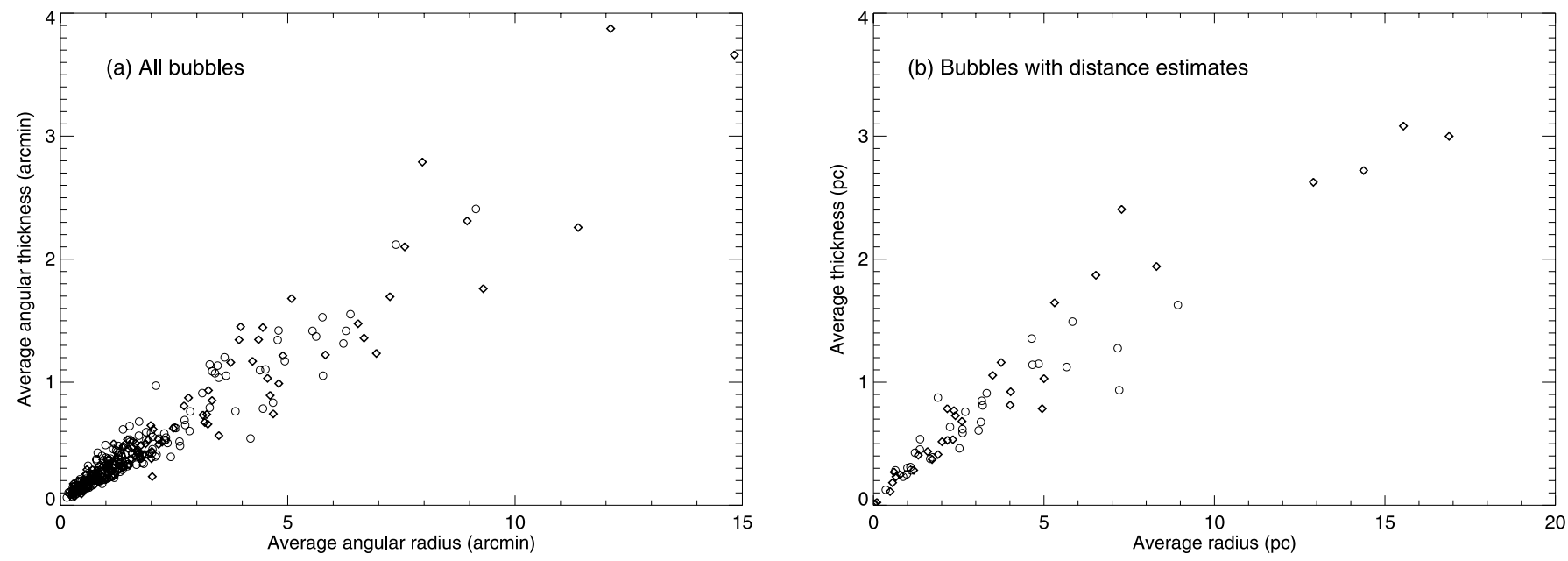

Fig. 11.-Observed average shell thickness $\langle T\rangle$ is linearly correlated with average radius $\langle R\rangle$ for $(a)$ all bubbles in our sample (angular units) and (b) the subset of bubbles for which we have kinematic distance estimates from $\mathrm{H}$ II regions (absolute units, calculated assuming near or tangent point distances). Diamonds and circles denote bubbles in the northern and southern halves, respectively, of the GLIMPSE survey area.

clusters. In this section we examine spatial coincidences of these objects with the bubbles in Tables 2 and 3 to determine what types of objects are primarily responsible for producing the observed bubbles.

The bubbles in the GLIMPSE sample are particularly bright in IRAC bands that contain PAH spectral features $(3.3 \mu \mathrm{m}$ in band 1 , $6.2 \mu \mathrm{m}$ in band 3, and 7.7 and $8.6 \mu \mathrm{m}$ in band 4$)$. Since PAHs are strongly excited in the neighborhood of young OB stars (Churchwell et al. 2004 and references therein), it is likely that many of the bubbles identified in the GLIMPSE survey will have counterparts with radio-identified $\mathrm{H}$ II regions (Cohen \& Green 2001) and/or star clusters.

$\mathrm{H}$ II regions. - We compared the Paladini et al. (2003) master catalog of radio-detected Galactic $\mathrm{H}$ II regions with our bubble catalog. Positions accurate to within a few arcseconds were generously provided by R. Paladini (2006, private communication). Out of 188 bubbles in the southern half of the survey, 39 contain the central position of an $\mathrm{H}$ II region within their angular radius. Bubbles occupy a combined area of $0.83 \mathrm{deg}^{2}$ or $0.7 \%$ of the southern plane survey area. Thus, out of the 313 radio $\mathrm{H}$ II regions

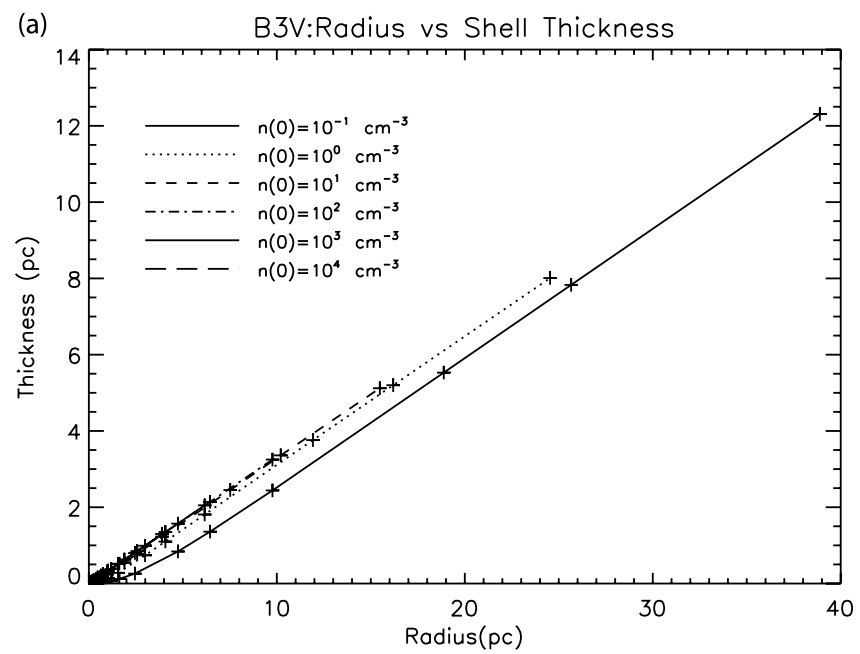

in the Paladini catalog that lie within the southern GLIMPSE survey area, we should find only 2 that are aligned by chance with bubbles. Out of 134 bubbles in the northern plane, we found 44 bubbles that contain the central position of an $\mathrm{H}$ II region within their radius. Bubbles occupy a total area of $1.29 \mathrm{deg}^{2}$ or $1.1 \%$ of the northern survey area, implying that out of the $391 \mathrm{H}$ II regions that lie in the northern plane survey area in the Paladini catalog, only 4 chance alignments are expected. Out of the total sample of 322 bubbles in the entire survey area, 83 (just over $25 \%$ ) have $\mathrm{H}$ II region central positions lying within the radius of a bubble. This represents a significant correlation between bubbles and Galactic $\mathrm{H}$ II regions.

Clusters. - We checked for coincidences (cluster centers lying within the radius of a bubble) with several lists of known optical, near-IR, and mid-IR star clusters. In the Mercer et al. (2005) catalog of 92 new clusters discovered using the GLIMPSE survey data, 3 clusters out of 25 are coincident with bubbles in the north and 17 out of 67 are coincident in the south. We also found 15 clusters out of 83 listed in the Dutra et al. (2003) near-IR catalog of southern clusters detected in the Two Micron All Sky

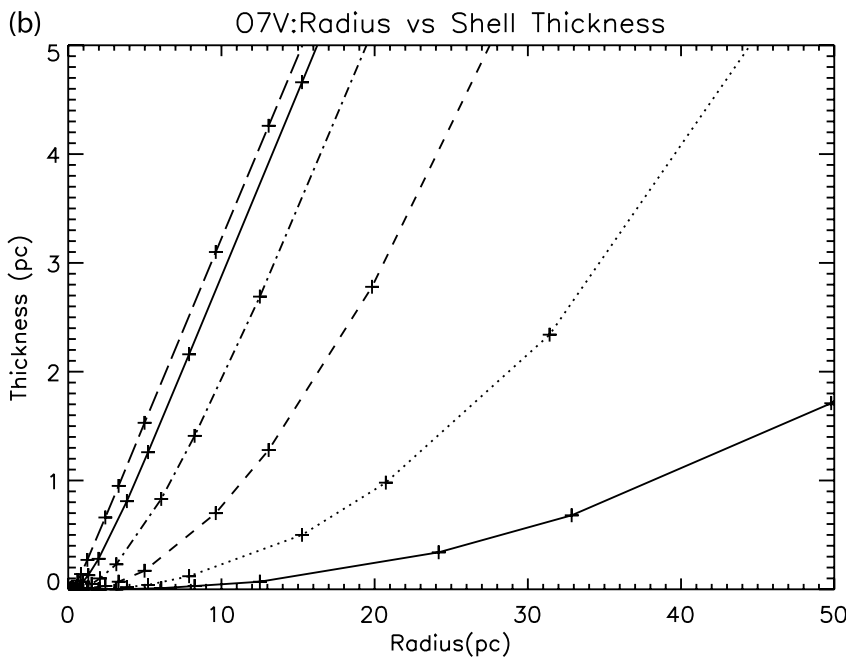

FIG. 12. - Shell thickness vs. radius predicted by the W77 models. Different curves are plotted for a wide range of ambient ISM densities surrounding bubbles blown by $(a)$ a B3 V star and $(b)$ an $\mathrm{O} 7 \mathrm{~V}$ star. 


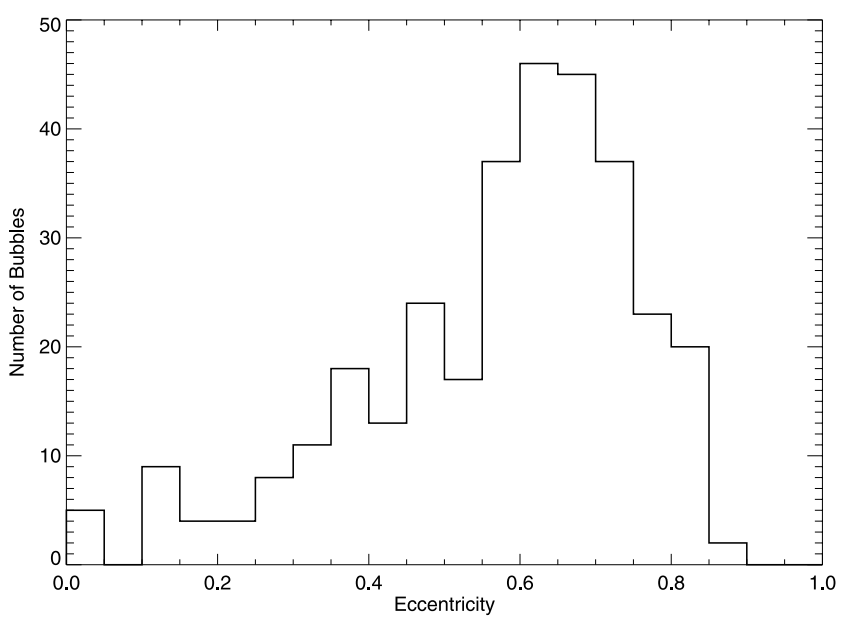

FIG. 13.-Distribution of bubble eccentricities. Note the large fraction of bubbles with relatively high eccentricities.

Survey (2MASS) that are located within bubbles in our sample. Six clusters out of 54 in the Bica et al. (2003) catalog of northern clusters detected in $2 \mathrm{MASS}$ are located within bubbles in our sample. The WEBDA ${ }^{14}$ catalog of nearby open clusters was also searched for coincidences, but only one cluster, vdBH 151, was found within a bubble (RCW 79; see Fig. 2b); this cluster was also included in the Dutra et al. (2003) catalog. In summary, out of a total of 229 known clusters lying in the GLIMPSE survey area, 41 are located within bubbles in our sample. The bubbles containing these 41 clusters are given the CC morphology flag in Tables 2 and $3 .{ }^{15}$ Based on occupied areas, we expect to find less than two chance overlaps of a cluster with a bubble in the entire survey. The fact that $13 \%$ of the bubbles in our sample are coincident with open clusters demonstrates a significant correlation between bubbles and open clusters.

SNRs.-Although SNRs have been detected in the GLIMPSE survey, they tend to be faint and have a wide range of mid-infrared colors. Of the 95 known SNRs within the GLIMPSE survey area, only 18 were identified by Reach et al. (2006) in the GLIMPSE survey. We also compared our bubble catalog with Green's 2004 catalog ${ }^{16}$ of SNRs and found only three radio SNRs whose center positions lie within the radius of a bubble. These are W44 (N63), W49B (N88), and G311.53-0.3 (S131). The angular sizes of these three SNRs are very similar to those of the bubbles with which their centers coincide. All three show strong emission in IRAC band 2 relative to the other IRAC bands, and this emission has distinctive filamentary structure. We therefore conclude that only these three SNRs correspond to bubbles in our sample.

Planetary nebulae.-We also tried to identify known planetary nebulae in the GLIMPSE survey with the bubbles in Tables 2 and 3 . None of the planetary nebulae listed in the Kerber et al. (2003) catalog that have counterparts in the GLIMPSE survey are identified with any of the bubbles in Tables 2 and 3. Also, none of the known W-R stars in the survey area have detected circumstellar bubbles in GLIMPSE (R. Gruendl 2006, private communication).

\footnotetext{
14 See http://www.univie.ac.at/webda/.

15 Tables 2 and 3 also contain 15 bubbles with the $\mathrm{CC}$ flag that were not found to correlate with known clusters. These designations were based solely on visual interpretation of the GLIMPSE images and hence the inferred clusters have not been included in our statistical analysis. If included, the correlation of bubbles with clusters would be $17 \%$.

16 See http://www.mrao.cam.ac.uk/surveys/snrs/snrs.data.html.
}

The location and size of bubbles in the GLIMPSE survey are plotted as solid circles in Figure 14, with the locations and sizes of $\mathrm{H}$ II regions plotted as dotted circles with plus signs denoting their central positions. The locations of open clusters in the Mercer et al. (2005), Dutra et al. (2003), and Bica et al. (2003) catalogs are indicated by crosses. Those bubbles that are SNRs are identified by diamonds at their central positions. Only those $\mathrm{H}$ II regions and clusters whose positions coincide with bubbles are plotted. As Figure 14 shows, $25 \%$ of the bubbles overlap with known $\mathrm{H}$ II regions and $13 \%$ overlap with cataloged star clusters. These are not disjoint sets: the majority of bubbles enclosing star clusters also contain $\mathrm{H}$ II regions, representing nearly $10 \%$ of the total sample of bubbles.

Many of the small bubbles may be associated either with solitary B4-B9 stars that are too cool to produce a detectable radio $\mathrm{H}$ II region but have strong enough winds to produce detectable dust shells or with compact clusters composed of low-mass stars whose combined stellar winds or bipolar outflows are strong enough to produce a detectable dust shell. In either case, the star or cluster must also create enough UV flux to excite PAH emission. About three-quarters of the members of our sample are probably produced by B4-B9 stars and about one-quarter are produced by $\mathrm{O}-\mathrm{B} 3$ stars. This is reasonably consistent with the numbers of O-B3 relative to B4-B9 stars (Cox 2000).

For unresolved $\mathrm{H}$ II regions at the detection limit in IRAC band $2(\sim 1 \mathrm{mJy})$, we predict a flux density in the $\mathrm{H} \alpha$ line of $\sim 26.4 \mathrm{mJy}(\sim 12.8 \mathrm{mag})$ for a temperature of $10^{4} \mathrm{~K}$ and density $10^{2}-10^{6} \mathrm{~cm}^{-3}$. Thus, it should be possible to detect bubbles that lie at the detection limit of IRAC band 2 or brighter with optical observations of the $\mathrm{H} \alpha$ line, if the extinction $A(\mathrm{H} \alpha) \leq 3$ mag or $A_{V} \leq 4 \mathrm{mag}$. Not all of the bubbles are necessarily dynamically formed by stellar winds: some may simply be the illuminated edges of $\mathrm{H}$ II regions where $\mathrm{PAH}$ features are excited by radiation from the $\mathrm{H}$ II region.

\section{BUBBLES WITH KNOWN DISTANCES}

Seventy-four of the $83 \mathrm{H}$ II regions whose centers lie within a bubble have measured radio recombination line radial velocities (Paladini et al. 2003). The Galactic rotation model of Brand \& Blitz (1993) with a distance of $R_{\odot}=8.5 \mathrm{kpc}$ to the Galactic center and a velocity $\Theta=220 \mathrm{~km} \mathrm{~s}^{-1}$ of the Sun around the Galactic center was used to determine kinematic distances to these $\mathrm{H}$ II regions. Table 4 lists the subset of 66 bubbles for which distances are determined from the radial velocities of the overlapping $\mathrm{H}$ II regions. As we have shown, the probability of chance alignments of $\mathrm{H}$ II regions with bubbles is quite small $(<1 \%)$, so the assumption that the bubbles lie at the same distance as the overlapping $\mathrm{H}$ II regions is a very good one. The near and far distances, with errors based on the assumption that departures from circular orbits are typically $\sim 10 \mathrm{~km} \mathrm{~s}^{-1}$, are given in columns (2) and (3) and (6) and (7) of Table 4. Usually bubbles at the far distance would be so far way that only the near distance is a realistic choice, given the bias against very distant bubbles ( $\operatorname{see} \S \S 2.1$ and 3.2). The average radii and thicknesses of the bubbles in parsecs are given in columns (4) and (5) and (8) and (9) for bubbles at the near and far distance, respectively. Of the 63 bubbles, 15 lie at the tangent point, and no far distance is given for them. For the rest of the bubbles, we give both the near and far distances but emphasize that the near distances are most likely the correct ones.

The distribution of near distances in Table 4 peaks at $4.2 \mathrm{kpc}$ with a range of $0.2-8.4 \mathrm{kpc}$. The average radius corresponding to the near distances is $3.3 \mathrm{pc}$ and ranges from $<0.1$ to $16.9 \mathrm{pc}$. The corresponding average shell thickness is $0.8 \mathrm{pc}$ and ranges 

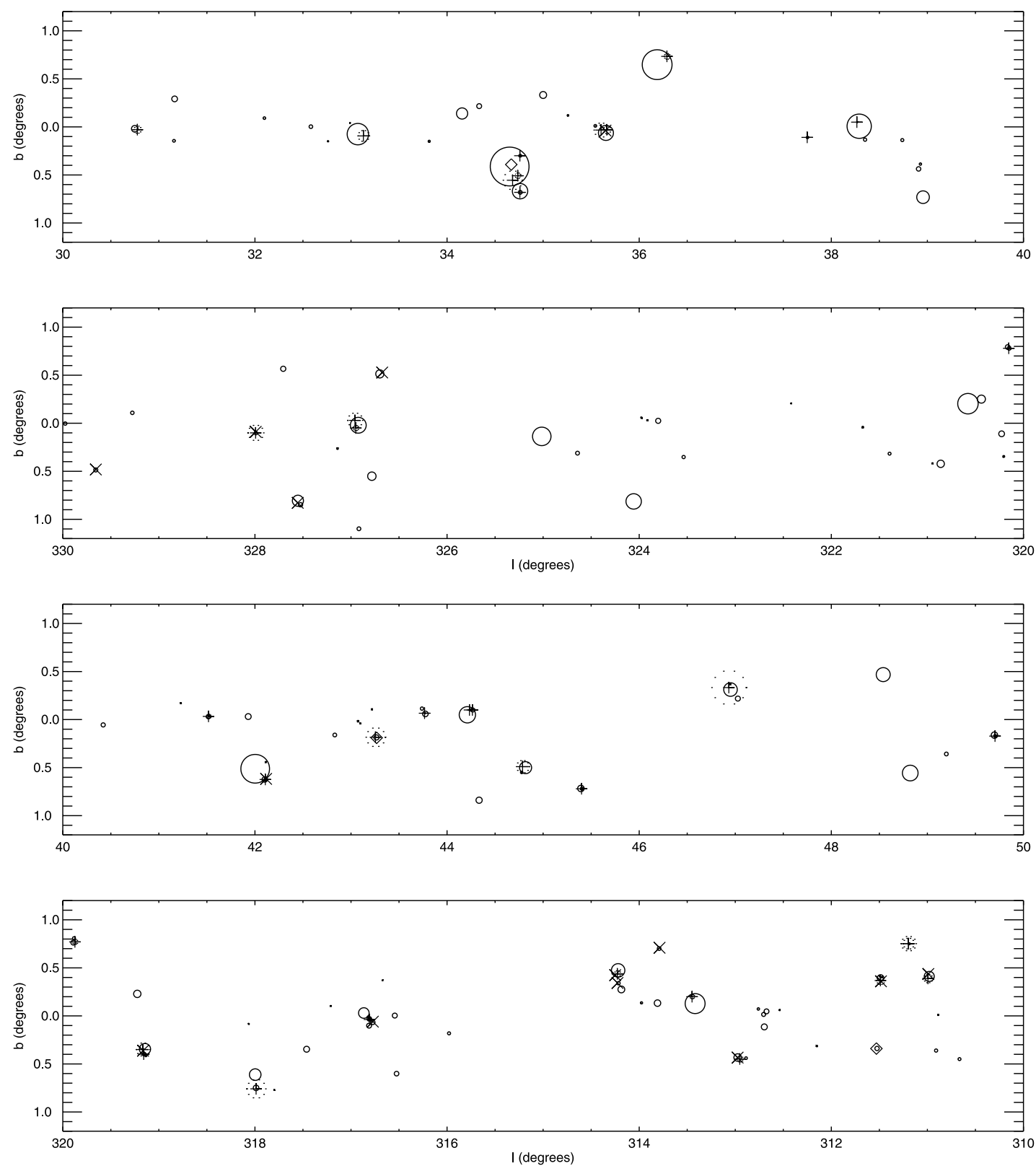

Fig. 14. - Distribution of bubbles with circles representing their angular sizes in the Galactic longitude range $30^{\circ}-50^{\circ}$ (in two $10^{\circ}$ segments) and $330^{\circ}-310^{\circ}$ (also in two $10^{\circ}$ segments). The four segments shown here represent about one-third of the full GLIMPSE survey area. Superimposed on the bubbles are the H in regions ( plus signs; surrounded by dotted circles representing the sizes of the $\mathrm{H}$ in regions) and stellar clusters (crosses). Those few bubbles that are actually SNRs are identified by diamonds at their central positions. [See the electronic edition of the Journal for additional color panels of this figure.]

from $<0.05$ to $3.9 \mathrm{pc}$. The observed radii and shell thicknesses are in reasonable agreement with the $\mathrm{W} 77$ predictions for a bubble of age $\geq 10^{6} \mathrm{yr}$ expanding into molecular clouds of density $\geq 10^{3} \mathrm{~cm}^{-3}$. The bubbles typically have thicknesses of about $25 \%$ of their radius. This is clearly much thicker than a simple shock front. The filamentary structure seen in many of the shells may indicate multiple shocks. There may also be a substantial region of swept-up ISM in front of the expanding shells.

\section{MULTIPLE BUBBLES}

Bubbles with $\mathrm{MB}$ designations (multiple bubbles) in column (11) of Tables 2 and 3 account for 22 out of 322 bubbles (7\%). The morphologies of these bubbles suggest that the expanding bubble surrounding an older star or star cluster has triggered the formation of one or more younger stars around the periphery of the expanding bubble. According to this hypothesis, star formation is triggered at the interface of the expanding bubble and the ambient ISM when preexisting, marginally stable molecular cores are overtaken by the expanding bubble, which provides enough external pressure to initiate collapse of the core. The secondgeneration star(s) form their own smaller bubbles that expand into the ISM, thereby producing the observed MB morphology.

Triggered star formation is an old idea that has been more frequently confronted by observations in recent years (Cohen et al. 2002; Zavagno et al. 2005 and references therein). In order for triggered star formation to be taken seriously, a parent bubble must remain visible long enough for the daughter bubble(s) to form and become visible. We see from Figure $11 b$ and Table 4 that most bubbles with known distances have radii between 1 and 9 pc. According to the W77 prescription, bubbles expand very 
rapidly when the central star first turns on nuclear burning, but the expansion decelerates rapidly as the bubble sweeps up surrounding material. After $\sim 10^{6} \mathrm{yr}$, an $\mathrm{O} 7 \mathrm{~V}$ star with a mass-loss rate of $3 \times 10^{-6} M_{\odot} \mathrm{yr}^{-1}$ and a wind terminal velocity of $1500 \mathrm{~km} \mathrm{~s}^{-1}$ will produce a bubble of radius $\lesssim 7.9$ pc with an ambient ISM density $\gtrsim 10^{3} \mathrm{~cm}^{-3}$, according to W77. A very young $\mathrm{O}$ star is likely to be surrounded by a molecular cloud density $\gtrsim 10^{3} \mathrm{~cm}^{-3}$ (Cesaroni et al. 1991 and others). A B3 V star is expected to produce a bubble of radius $\leq 1.6 \mathrm{pc}$ after $10^{6} \mathrm{yr}$ using the W77 prescription. Thus, it is reasonable to assign ages older than $10^{6} \mathrm{yr}$ to the potential parent bubbles observed (those with MB designations), the majority of which have radii greater than $3 \mathrm{pc}$ and less than $10 \mathrm{pc}$ (consider, for example, bubbles S133 and S145 in Figs. $2 a$ and $2 b$ and their entries in Table 4 ). The exact age-radius relation depends on the spectral type of the central star(s). The timescale for $\mathrm{O}$ star formation is on the order of a few times $10^{4} \mathrm{yr}$ based on inferred accretion rates (see Stahler \& Palla 2004 and references therein). Thus, it is possible for several episodes of triggered star formation to occur while the parent bubble is still visible. Additional observational support for triggered star formation can be obtained by measuring the relative ages of the stars giving rise to the parent and daughter bubbles in MB complexes by modeling their spectral energy distributions. This is a project in progress. Relative expansion velocities of all of the bubbles in a given MB complex also need to be measured. The fact that only $7 \%$ of the bubbles are candidate sites for triggered star formation indicates that this star formation mechanism is not a dominant one. However, it occurs often enough that it merits further study.

\section{SUMMARY}

A search by visual inspection of the GLIMPSE survey has revealed 322 full or partial rings that we propose represent complete or partial three-dimensional bubbles. Only three of the bubbles are identified with SNRs, and none with planetary nebulae or W-R stars. Eighty-three bubbles are associated with known $\mathrm{H}$ II regions, and 30 of these also contain cataloged near- or mid-IR stellar clusters. The $25 \%$ of bubbles that overlap known $\mathrm{H}$ II regions appear to be produced by stellar winds and radiation pressure from young OB stars in massive star formation regions. Dynamically formed bubbles in our sample that are bright at mid-infrared wavelengths but not coincident with SNRs, planetary nebulae, or radio $\mathrm{H}$ II regions require a star or cluster with enough UV to excite the PAH bands and a strong enough wind to evacuate a dust-free cavity around the star or star cluster. Stars that can do this and not produce detectable $\mathrm{H}$ II regions are B stars later than B3. Three-quarters of the bubbles in our sample do not coincide with known radio $\mathrm{H}$ in regions. We suggest that these are produced by B stars cooler than about B3. Presumably the 11 cataloged clusters lying within bubbles that are not associated with any detected $\mathrm{H}$ II region do not contain any star of type B3 or earlier.

We find that $24 \mu \mathrm{m}$ emission is systematically confined to the central regions of the bubbles where $8 \mu \mathrm{m}$ emission is absent. The $8 \mu \mathrm{m}$ emission is strong in the shells that define the bubbles and often extends outside the bubbles. We postulate that the $24 \mu \mathrm{m}$ emission is produced by thermal emission from hot dust with about a $10 \%$ contribution from small grains transiently heated by nearby OB stars (Li \& Draine 2001), whereas the $8 \mu \mathrm{m}$ emission is dominated by PAH features excited by softer radiation in the shell and beyond, in the PDRs of the bubbles. The observed peaking of $24 \mu \mathrm{m}$ emission at the centers of bubbles is puzzling. How can the dust survive in the near vicinity of
OB stars? Why is the dust not evacuated from the central regions by the winds and radiation pressure of the central OB stars? Static bubbles might be consistent with the observations, but this seems inconsistent with all that is known about OB star winds and radiation. These are open questions that must be left for further investigation.

Bubbles are strongly concentrated to the Galactic midplane with an angular scale height of $0.63 \pm 0.03$. Because the number of detected bubbles drops sharply as a function of distance for $D \gtrsim 4.2 \mathrm{kpc}$, we can estimate a value of $44 \mathrm{pc}$ corresponding to 0.6 at $4.2 \mathrm{kpc}$ for the physical scale height of bubbles in the Galactic plane. The peak of the latitude distribution is located about $0.08 \pm 0.03$ below the midplane. Since the distribution of all stars in the GLIMPSE survey is centered at $b=0^{\circ}$ (Benjamin et al. 2005), the distribution of stars that excite bubbles is slightly offset from the rest of the stars in the GLIMPSE catalog, as are radio $\mathrm{H}$ II regions and $\mathrm{CO}$ clouds.

The distribution of average bubble angular diameters peaks between $1^{\prime}$ and $3^{\prime}$. Over $98 \%$ of the bubbles are smaller than $10^{\prime}$, and almost $88 \%$ are smaller than $4^{\prime}$. The largest bubbles, almost without exception, are rather faint and located at large angular distances from the Galactic center where the background brightness is low. The same bubbles in the inner part of the Galaxy probably would not have been detected.

A large fraction of the bubbles are highly eccentric. About $65 \%$ have eccentricities $0.55 \leq e \leq 0.85$. The distribution peaks at $e \approx 0.65$. Only about $5 \%$ have $e<0.2$, and only $\approx 7 \%$ have $e>0.8$.

The bubble shells tend to be filamentary with little or no dust between the filaments, indicating that the filling factors of the bubble shells may be quite low. The maximum projected angular thickness of the bubbles is $<4^{\prime}$. Over $85 \%$ of the bubbles have thicknesses less than $1^{\prime}$, and over $97 \%$ have thicknesses less than $2^{\prime}$. About $90 \%$ of the bubbles have thicknesses between 0.2 and 0.4 of their outer radii.

Triggered star formation is suggested by the multiple bubble morphology of $7 \%$ of our sample.

On average, about 1.5 bubbles are found per square degree in the GLIMPSE survey with little dependence on longitude. The apparent density does not increase noticeably closer to the Galactic center because the detection rate for bubbles in GLIMPSE peaks at a distance of $4.2 \mathrm{kpc}$ within a horizon of $8 \mathrm{kpc}$. In general, it is difficult to identify very small and very large bubbles against a bright and spatially variable infrared background. Hence, the number of bubbles found in this study is almost certainly an underestimate of the actual number of bubbles in the surveyed area of the Galactic disk. The frequency of 1.5 bubbles per square degree and a scale height of $44 \mathrm{pc}$ at $4.2 \mathrm{kpc}$ together imply that bubbles blown by massive star formation regions (OB associations) occur with a minimum volume density of $200 \mathrm{kpc}^{-3}$ in the inner Galaxy.

We thank Stephan Jansen, who has been tireless in keeping our computers online. We also acknowledge the IRAC instrument team, who delivered such a superb instrument to Spitzer, and the staff of the Spitzer Science Center, who have been very supportive of the GLIMPSE program. We thank the anonymous referee, whose incisive comments led to the substantial improvement of this work. This research made use of Montage, funded by the National Aeronautics and Space Administration's Earth Science Technology Office, Computational Technologies Project, under Cooperative Agreement NCC5-626 between NASA and 
the California Institute of Technology. Support for this work, part of the Spitzer Space Telescope Legacy Science Program, was provided by NASA through contracts 1224653 (University of Wisconsin at Madison), 1225025 (Boston University), 1224681 (University of Maryland), 1224988 (Space Science Institute), 1242593 (University of California at Berkeley), 1253153 (Uni- versity of Minnesota), 11253604 (University of Wyoming), and 1256801 (University of Wisconsin at Whitewater) by the Jet Propulsion Laboratory, California Institute of Technology under NASA contract 1407 .

\section{Facilities: Spitzer(IRAC)}

Abbott, D. C., Bieging, J. H., \& Churchwell, E. 1981, ApJ, 250, 645

Becker, R. H, White, R. L., McLean, B. J., Helfand, D. J., \& Zoonematkermani, S. 1990, ApJ, 358, 485

Benjamin, R. A., et al. 2003, PASP, 115, 953 2005, ApJ, 630, L149

Bica, E., Dutra, C. M., Soares, J., \& Barbuy, B. 2003, A\&A, 404, 223

Brand, J., \& Blitz, L. 1993, A\&A, 275, 67

Burton, W. B. 1985, A\&AS, 62, 365

Cesaroni, R., Walmsley, C. M., Koempe, C., \& Churchwell, E. 1991, A\&A, 252,278

Churchwell, E., et al. 2004, ApJS, 154, 322

Clemens, D. P., Sanders, D. B., \& Scoville, N. Z. 1988, ApJ, 327, 139

Cohen, M., \& Green, A. J. 2001, MNRAS, 325, 531

Cohen, M., Green, A. J., Parker, Q. A., Mader, S., \& Cannon, R. D. 2002, MNRAS, 336, 736

Cohen, R. S., \& Thaddeus, P. 1977, ApJ, 217, L155

Cox, A. N., ed. 2000, Allen's Astrophysical Quantities (4th ed.; New York: AIP)

Dame, T. M., \& Thaddeus, P. 1985, ApJ, 297, 751

Dame, T. M., et al. 1987, ApJ, 322, 706

Dutra, C. M., Bica, E., Soares, J., \& Barbuy, B. 2003, A\&A, 400, 533

Fazio, G. G., et al. 2004, ApJS, 154, 10

Gilmore, G. F., \& Zeilik, M. 2000, in Allen's Astrophysical Quantities, ed. A. N. Cox (New York: AIP), 479

Haffner, L. M., Reynolds, R. J., Tufte, S. L., Madsen, G. J., Jaehnig, K. P., \& Percival, J. W. 2003, ApJS, 149, 405

\section{REFERENCES}

Helfand, D. J., Zoonematkermani, S., Becker, R. H., \& White, R. L. 1992, ApJS, 80, 211

Hollenbach, D., Chu, S.-I., \& McCray, R. 1976, ApJ, 208, 458

Hollenbach, D. J., \& Tielens, A. G. G. M. 1997, ARA\&A, 35, 179

Kerber, F., Mignani, R. P., Guglielmetti, F., \& Wicenec, A. 2003, A\&A, 408, 1029

Kerr, F. J., Bowers, P. F., Jackson, P. D., \& Kerr, M. 1986, A\&AS, 66, 373

Li, A., \& Draine, B. T. 2001, ApJ, 550, L213

McClure-Griffiths, N. M., Dickey, J. M., Gaensler, B. M., \& Green, A. J. 2002, ApJ, 578, 176

Mercer, E. P., et al. 2005, ApJ, 635, 560

Paladini, R., Burigana, C., Davies, R. D., Maino, D., Bersanelli, M., Cappellini, B., Platania, P., \& Smoot, G. 2003, A\&A, 397, 213

Paladini, R., Davies, R. D., \& DeZotti, G. 2004, MNRAS, 347, 237

Price, S. D., Egan, M. P., Carey, S. J., Mizuno, D. R., \& Kuchar, A. 2001, AJ, 121, 2819

Reach, W. T., et al. 2006, AJ, 131, 1479

Stahler, S. W., \& Palla, S. 2004, The Formation of Stars (New York: Wiley)

Vacca, W. D., Garmany, C. D., \& Shull, M. J. 1996, ApJ, 460, 914

Weaver, R., McCray, R., Castor, J., Shapiro, P., \& Moore, R. 1977, ApJ, 218 , 377 (W77)

Werner, M. W., et al. 2004, ApJS, 154, 1

Wood, D. O. S., \& Churchwell, E. 1989, ApJ, 340, 265

Zavagno, A., Deharveng, L., Brand, J., Massi, F., Caplan, J., Comerón, F., \& Lefloch, B. 2005, in IAU Symp. 227, Massive Star Birth: A Crossroads of Astrophysics, ed. R. Cesaroni et al. (Cambridge: Cambridge Univ. Press), 346 